Annals of Mathematics 00 (XXXX), 1-40

http://dx.doi.org/10.4007/annals.XXXX.00.0.0000

\title{
Measurable circle squaring
}

\author{
By Łukasz Grabowski, András Máthé, and Oleg Pikhurko
}

\begin{abstract}
Laczkovich proved that if bounded subsets $A$ and $B$ of $\mathbb{R}^{k}$ have the same non-zero Lebesgue measure and the upper box dimension of the boundary of each set is less than $k$, then there is a partition of $A$ into finitely many parts that can be translated to form a partition of $B$. Here we show that it can be additionally required that each part is both Baire and Lebesgue measurable. As special cases, this gives measurable and translation-only versions of Tarski's circle squaring and Hilbert's third problem.
\end{abstract}

\section{Introduction}

We call two sets $A, B \subseteq \mathbb{R}^{k}$ equidecomposable and denote this as $A \sim B$ if there are a partition $A=A_{1} \cup \ldots \cup A_{n}$ (into finitely many parts) and isometries $\gamma_{1}, \ldots, \gamma_{n}$ of $\mathbb{R}^{k}$ such that the images of the parts $\gamma_{1}\left(A_{1}\right), \ldots, \gamma_{n}\left(A_{n}\right)$ partition $B$. In other words, we can cut $A$ into finitely many pieces and rearrange them to form the set $B$. When this can be done is a very basic question that one can ask about two sets and, as Dubins, Hirsch, and Karush [6, Page 239] write, "variants of the problems studied here already occur in Euclid". We refer the reader to various surveys and expositions of this area $([8,9,11,19,20,21,39])$ as well as the excellent book by Tomkowicz and Wagon [38].

The version that is closest to our everyday intuition (e.g. via puzzles like "Tangram", "Pentomino", or "Eternity") is perhaps the dissection congruence in $\mathbb{R}^{2}$ where the pieces have to be polygonal and their boundary can be ignored when taking partitions. A well-known example from elementary mathematics is finding the area of a triangle by dissecting it into a rectangle. In fact, as

Ł.G. was partially supported by EPSRC grant EP/K012045/1 and by Fondations Sciences Mathématiques de Paris during the programme Marches Aléatoires et Géométrie Asymptotique des Groupes at Institut Henri-Poincaré. A.M. was partially supported by a Levehulme Trust Early Career Fellowship and by the Hungarian National Research, Development and Innovation Office - NKFIH, 104178. O.P. was partially supported by ERC grant 306493 and EPSRC grant EP/K012045/1. 
it was discovered around 1832 independently by Bolyai and Gerwien, any two polygons of the same area are congruent by dissections. (Apparently, Wallace proved this result already in 1807; see [38, Pages 34-35] for a historical account and further references.) The equidecomposition problem for polygons is also completely resolved: a result of Tarski [34] (see e.g. [38, Theorem 3.9]) gives that any two polygons of the same area are equidecomposable.

Banach and Tarski [3] proved that, in dimensions 3 or higher, any two bounded sets with non-empty interior are equidecomposable; in particular, we get the famous Banach-Tarski Paradox that a ball can be doubled. On the other hand, as it was also shown in [3] by using the earlier results of Banach [2], a ball in $\mathbb{R}^{k}$ cannot be doubled for $k=1,2$. This prompted von Neumann [27] to investigate what makes the cases $k=1,2$ different using the group-theoretic point of view, which started the study of amenable groups.

Around that time, Tarski [35] asked if the disk and square in $\mathbb{R}^{2}$ of the same area are equidecomposable, which became known as Tarski's circle squaring. Von Neumann [27] showed that circle squaring is possible if arbitrary measure-preserving affine transformations are allowed. On the other hand, some negative evidence was provided by Dubins, Hirsch, and Karush [6] who showed that a circle and a square are not scissor congruent (when the pieces are restricted to be topological disks and their boundary can be ignored) and by Gardner [7] who proved that circle-squaring is impossible if we use a locally discrete subgroup of isometries of $\mathbb{R}^{2}$. However, the deep paper of Laczkovich [15] showed that the answer to Tarski's question is affirmative. In fact, his main result (coming from the papers $[15,16,17]$ ) is much more general and stronger. In order to state it, we need some definitions.

We call two sets $A, B \subseteq \mathbb{R}^{k}$ equivalent (and denote this by $A \stackrel{\operatorname{Tr}}{\sim} B$ ) if they are equidecomposable using translations, that is, there are partitions $A=A_{1} \cup \ldots \cup A_{m}$ and $B=B_{1} \cup \ldots \cup B_{m}$, and vectors $\boldsymbol{v}_{1}, \ldots, \boldsymbol{v}_{m} \in \mathbb{R}^{k}$ such that $B_{i}=A_{i}+\boldsymbol{v}_{i}$ for each $i \in\{1, \ldots, m\}$. Let $\lambda=\lambda_{k}$ denote the Lebesgue measure on $\mathbb{R}^{k}$. The box (or grid, or upper Minkowski) dimension of $X \subseteq \mathbb{R}^{k}$ is

$$
\operatorname{dim}_{\square}(X):=k-\liminf _{\varepsilon \rightarrow 0^{+}} \frac{\log \lambda\left(\left\{\boldsymbol{x} \in \mathbb{R}^{k}: \operatorname{dist}(\boldsymbol{x}, X) \leqslant \varepsilon\right\}\right)}{\log \varepsilon},
$$

where $\operatorname{dist}(\boldsymbol{x}, X)$ means e.g. the $L^{\infty}$-distance from the point $\boldsymbol{x}$ to the set $X$. Let $\partial X$ denote the topological boundary of $X$. It is easy to show that if $A \subseteq \mathbb{R}^{k}$ satisfies $\operatorname{dim}_{\square}(\partial A)<k$, then $A$ is Lebesgue measurable and, furthermore, $\lambda(A)>0$ if and only if $A$ has non-empty interior. With these observations, the result of Laczkovich can be formulated as follows.

Theorem 1.1 (Laczkovich $[15,16,17])$. Let $k \geqslant 1$ and let $A, B \subseteq \mathbb{R}^{k}$ be bounded sets with non-empty interior such that $\lambda(A)=\lambda(B), \operatorname{dim}_{\square}(\partial A)<k$, and $\operatorname{dim}_{\square}(\partial B)<k$. Then $A$ and $B$ are equivalent. 
Theorem 1.1 applies to circle squaring since the boundary of each of these sets has box dimension 1 . As noted in [16], the inequality $\operatorname{dim}_{\square}(\partial A)<k$ holds if $A \subseteq \mathbb{R}^{k}$ is a convex bounded set or if $A \subseteq \mathbb{R}^{2}$ has connected boundary of finite linear measure; thus Theorem 1.1 applies to such sets as well.

Note that the condition that $\lambda(A)=\lambda(B)$ is necessary in Theorem 1.1. Indeed, the group of translations of $\mathbb{R}^{k}$ is amenable (since it is an Abelian group) and therefore the Lebesgue measure on $\mathbb{R}^{k}$ can be extended to a translationinvariant finitely additive measure defined on all subsets (and so equivalent sets which are measurable must necessarily have the same measure); see e.g. [38, Chapter 12] for a detailed discussion. Laczkovich [18] showed that one cannot replace the box dimension with the Hausdorff dimension in Theorem 1.1; see also [22] for further examples of non-equivalent sets.

The proof of Theorem 1.1 by Laczkovich directly relies on the Axiom of Choice in a crucial way. Thus the pieces that he obtains need not be measurable. Laczkovich [15, Section 10] writes: "The problem whether or not the circle can be squared with measurable pieces seems to be the most interesting."

This problem remained open until now, although some modifications of it were resolved. Henle and Wagon (see [38, Theorem 9.3]) showed that, for any $\varepsilon>0$, one can square a circle with Borel pieces if one is allowed to use similarities of the plane with scaling factor between $1-\varepsilon$ and $1+\varepsilon$. Pieces can be made even more regular if some larger class of maps can be used (such as arbitrary similarities or affine maps), see e.g. [11, 31, 32, 33]. Also, if countably many pieces are allowed, then a simple measure exhaustion argument shows that, up to a nullset, one can square a circle with measurable pieces (see [3, Theorem 41] or [38, Theorem 11.26]); the error nullset can be then eliminated by e.g. applying Theorem 1.1 .

The authors of this paper prove in [10] that every two bounded measurable sets $A, B \subseteq \mathbb{R}^{k}, k \geqslant 3$, with non-empty interior and of the same measure are equidecomposable with Lebesgue measurable pieces. In particular, this gives a measurable version of Hilbert's third problem (as asked by Wagon [40, Question 3.14]): one can split a regular tetrahedron into finitely many measurable pieces and rearrange them into a cube. These results rely on the spectral gap property of the natural action of $S O(k)$ on the $(k-1)$-dimensional sphere in $\mathbb{R}^{k}$ for $k \geqslant 3$ and do not apply when $k \leqslant 2$. Also, the equidecompositions obtained in [10] cannot be confined to use translations only.

Here we fill a part of this gap. Namely, our main main result (Theorem 1.2) shows that it can be additionally required in Theorem 1.1 that all pieces are Lebesgue measurable.

In fact, Theorem 1.2 gives pieces that are also Baire measurable (or Baire for short), that is, each one is the symmetric difference of a Borel set and a meagre set. The study of equidecompositions with Baire sets was largely 
motivated by Marczewski's problem from 1930 whether $\mathbb{R}^{k}$ admits a non-trivial isometry-invariant finitely additive Borel measure that vanishes on bounded meagre sets. It is not hard to show that the answer is positive for $k \leqslant 2$, see e.g. [38, Corollary 13.3]. However, the problem for $k \geqslant 3$ remained open for over 60 years until it was resolved in the negative by Dougherty and Foreman $[4,5]$ who proved in particular that any two bounded Baire sets $A, B \subseteq \mathbb{R}^{k}$ with non-empty interior are equidecomposable with Baire measurable pieces (and thus a ball can be doubled with Baire pieces). A short and elegant proof of a more general result was recently given by Marks and Unger [26] (see also [12]). However, as noted in [26, Page 406], the problem whether circle squaring is possible with Baire measurable parts remained open. Also, the results in $[4,5,26]$ do not apply to the translation equidecomposability $\stackrel{\operatorname{Tr}}{\sim}$, even in higher dimensions. Here we resolve these questions in the affirmative, under the assumptions of Theorem 1.1:

Theorem 1.2. Let $k \geqslant 1$ and let $A, B \subseteq \mathbb{R}^{k}$ be bounded sets with nonempty interior such that $\lambda(A)=\lambda(B), \operatorname{dim}_{\square}(\partial A)<k$, and $\operatorname{dim}_{\square}(\partial B)<k$. Then $A \stackrel{\text { Tr }}{\sim} B$ with parts that are both Baire and Lebesgue measurable.

In addition to implying measurable translation-only versions of Tarski's circle squaring, Hilbert's third problem, and Wallace-Bolyai-Gerwien's theorem (a question of Laczkovich [15, Page 114]), Theorem 1.2 also disproves the following conjecture of Gardner [8, Conjecture 5] for all $k \geqslant 2$.

Conjecture 1.3. Let $P$ be a polytope and $K$ a convex body in $\mathbb{R}^{k}$. If $P$ and $K$ are equidecomposable with Lebesgue measurable pieces under the isometries from an amenable group, then $P$ and $K$ are equidecomposable with convex pieces under the same isometries.

Indeed, for example, let $P$ be a cube and $K$ be a ball of the same volume. It is not hard to show directly that $K$ and $P$ are not equidecomposable with convex pieces, even under the groups of all isometries of $\mathbb{R}^{k}$ for $k \geqslant 2$. Since Theorem 1.2 uses only translations (that form an amenable group), Conjecture 1.3 is false.

This paper is organised as follows. In Section 2 we reduce the problem to the torus $\mathbb{T}^{k}:=\mathbb{R}^{k} / \mathbb{Z}^{k}$ and state a sufficient condition for measurable equivalence in Theorem 2.2. We also describe there how Theorem 1.2 can be deduced from Theorem 2.2, using some results of Laczkovich [16]. The main bulk of this paper consists of the proof of Theorem 2.2 in Sections 4 and 5. These sections are dedicated to respectively Lebesgue and Baire measurability (while some common definitions and auxiliary results are collected in Section 3). We organised the presentation so that Sections 4 and 5 can essentially be read independently of each other. Section 6 contains some concluding remarks. 
In order to avoid ambiguities, a closed (resp. half-open) interval will always mean an interval of integers (resp. reals); thus, for example, $[m, n]:=$ $\{m, m+1, \ldots, n\} \subseteq \mathbb{Z}$ while $[a, b):=\{x \in \mathbb{R}: a \leqslant x<b\}$. Also, we denote $[n]:=\{1, \ldots, n\}$ and $\mathbb{N}:=\{0,1,2, \ldots\}$.

\section{Sufficient condition for measurable equivalence}

The $k$-dimensional torus $\mathbb{T}^{k}$ is the quotient of the Abelian group $\left(\mathbb{R}^{k},+\right)$ by the subgroup $\left(\mathbb{Z}^{k},+\right)$. We identify $\mathbb{T}^{k}$ with the real cube $[0,1)^{k}$, endowed with the addition of vectors modulo 1 .

By scaling the bounded sets $A, B \subseteq \mathbb{R}^{k}$ by the same factor and translating them, we can assume that they are subsets of $[0,1)^{k}$. Note that if $A, B \subseteq$ $[0,1)^{k}$ are (measurably) equivalent with translations taken modulo 1 , then they are (measurably) equivalent in $\mathbb{R}^{k}$ as well using at most $2^{k}$ times as many translations. (In fact, if each of $A, B$ has diameter less than $1 / 2$ with respect to the $L^{\infty}$-distance, then we do not need to increase the number of translations at all.) So we work inside the torus from now on.

Suppose that we have fixed some vectors $\boldsymbol{x}_{1}, \ldots, \boldsymbol{x}_{d} \in \mathbb{T}^{k}$ that are free, that is, no non-trivial integer combination of them is the zero element of $\left(\mathbb{T}^{k},+\right)$ (or, equivalently, $\boldsymbol{x}_{1}, \ldots, \boldsymbol{x}_{d}, \boldsymbol{e}_{1}, \ldots, \boldsymbol{e}_{k}$, when viewed as vectors in $\mathbb{R}^{k}$, are linearly independent over the rationals, where $\boldsymbol{e}_{1}, \ldots, \boldsymbol{e}_{k}$ are the standard basis vectors of $\mathbb{R}^{k}$ ).

When reading the following definitions (many of which implicitly depend on $\boldsymbol{x}_{1}, \ldots, \boldsymbol{x}_{d}$ ), the reader is advised to keep in mind the following connection to Theorems 1.1 and 1.2: we fix some large integer $M$ and try to establish the equivalence $A \stackrel{\operatorname{Tr}}{\sim} B$ by translating only by vectors from the set

$$
\mathcal{V}_{M}:=\left\{n_{1} \boldsymbol{x}_{1}+\ldots+n_{d} \boldsymbol{x}_{d}: \boldsymbol{n} \in \mathbb{Z}^{d},\|\boldsymbol{n}\|_{\infty} \leqslant M\right\} .
$$

Thus, if we are successful, then the total number of pieces is at most $\left|\mathcal{V}_{M}\right|=$ $(2 M+1)^{d}$.

By a coset of $\boldsymbol{u} \in \mathbb{T}^{k}$ we will mean the coset taken with respect to the subgroup of $\left(\mathbb{T}^{k},+\right)$ generated by $\boldsymbol{x}_{1}, \ldots, \boldsymbol{x}_{d}$, that is, the set $\left\{\boldsymbol{u}+\sum_{j=1}^{d} n_{j} \boldsymbol{x}_{j}\right.$ : $\left.\boldsymbol{n} \in \mathbb{Z}^{d}\right\} \subseteq \mathbb{T}^{k}$. For $X \subseteq \mathbb{T}^{k}$, we define

$$
X_{\boldsymbol{u}}:=\left\{\boldsymbol{n} \in \mathbb{Z}^{d}: \boldsymbol{u}+n_{1} \boldsymbol{x}_{1}+\ldots+n_{d} \boldsymbol{x}_{d} \in X\right\} .
$$

Informally speaking, $X_{\boldsymbol{u}} \subseteq \mathbb{Z}^{d}$ records which elements of the coset of $\boldsymbol{u} \in \mathbb{T}^{k}$ are in $X$.

If, for every $\boldsymbol{u} \in \mathbb{T}^{k}$, we have a bijection $\mathcal{M}_{\boldsymbol{u}}: A_{\boldsymbol{u}} \rightarrow B_{\boldsymbol{u}}$ such that

$$
\left\|\mathcal{M}_{\boldsymbol{u}}(\boldsymbol{n})-\boldsymbol{n}\right\|_{\infty} \leqslant M, \quad \text { for all } \boldsymbol{n} \in A_{\boldsymbol{u}},
$$

then Theorem 1.1 follows. Indeed, using the Axiom of Choice select a set $U \subseteq \mathbb{T}^{k}$ that intersects each coset in precisely one element. Now, each $\boldsymbol{a} \in A$ 
can be uniquely written as $\boldsymbol{u}+\sum_{j=1}^{d} n_{j} \boldsymbol{x}_{j}$ with $\boldsymbol{u} \in U$ and $\boldsymbol{n} \in \mathbb{Z}^{d}$; if we assign $\boldsymbol{a}$ to the piece which is translated by the vector $\sum_{j=1}^{d}\left(m_{j}-n_{j}\right) \boldsymbol{x}_{j}$ where $\boldsymbol{m}:=\mathcal{M}_{\boldsymbol{u}}(\boldsymbol{n})$, then we get the desired equivalence $A \stackrel{\operatorname{Tr}}{\sim} B$. This reduction was used by Laczkovich [15, 16, 17]; of course, the main challenge he faced was establishing the existence of the bijections $\mathcal{M}_{\boldsymbol{u}}$ as in (2). Here, in order to prove Theorem 1.2 , we will additionally need that the family $\left(\mathcal{M}_{\boldsymbol{u}}\right)_{\boldsymbol{u} \in \mathbb{T}^{k}}$ is consistent for different choices of $\boldsymbol{u}$ and gives measurable parts.

By an $n$-cube $Q \subseteq \mathbb{Z}^{d}$ we mean the product of $d$ intervals in $\mathbb{Z}$ of size $n$, i.e. $Q=\prod_{j=1}^{d}\left[n_{j}, n_{j}+n-1\right]$ for some $\left(n_{1}, \ldots, n_{d}\right) \in \mathbb{Z}^{d}$. If $n$ is an integer power of 2 , we will call the cube $Q$ binary. Given a function $\Phi:\left\{2^{i}: i \in \mathbb{N}\right\} \rightarrow \mathbb{R}$ and a real $\delta \geqslant 0$, a set $X \subseteq \mathbb{Z}^{d}$ is called $\Phi$-uniform (of density $\delta$ ) if, for every $i \in \mathbb{N}$ and $2^{i}$-cube $Q \subseteq \mathbb{Z}^{d}$, we have that

$$
|| X \cap Q|-\delta| Q|| \leqslant \Phi\left(2^{i}\right)
$$

In other words, this definition says that the discrepancy with respect to binary cubes between the counting measure of $X$ and the measure of constant density $\delta$ is upper bounded by $\Phi$. A set $Y \subseteq \mathbb{T}^{k}$ is called $\Phi$-uniform (of density $\delta$ with respect to $\left.\boldsymbol{x}_{1}, \ldots, \boldsymbol{x}_{d}\right)$ if $Y_{\boldsymbol{u}}$ is $\Phi$-uniform of density $\delta$ for every $\boldsymbol{u} \in \mathbb{T}^{k}$.

These notions are of interest to us because of the following sufficient condition for $A \stackrel{\operatorname{Tr}}{\sim} B$ that directly follows from Theorems 1.1 and 1.2 in Laczkovich [17].

Theorem 2.1 (Laczkovich [17]). Let $k, d \geqslant 1$ be integers, let $\delta>0$, let $\boldsymbol{x}_{1}, \ldots, \boldsymbol{x}_{d} \in \mathbb{T}^{k}$ be free, let a function $\Phi:\left\{2^{i}: i \in \mathbb{N}\right\} \rightarrow \mathbb{R}$ satisfy

$$
\sum_{i=0}^{\infty} \frac{\Phi\left(2^{i}\right)}{2^{(d-1) i}}<\infty,
$$

and let sets $A, B \subseteq \mathbb{T}^{k}$ be $\Phi$-uniform of density $\delta$ with respect to $\boldsymbol{x}_{1}, \ldots, \boldsymbol{x}_{d}$. Then $A \stackrel{T r}{\sim} B$, using translations that are integer combinations of the vectors $\boldsymbol{x}_{j}$.

Roughly speaking, the condition (4) states that the discrepancy of $A_{\boldsymbol{u}}$ and $B_{\boldsymbol{u}}$ with respect to any $2^{i}$-cube $Q$ decays noticeably faster than the size of the boundary of $Q$ as $i \rightarrow \infty$. On the other hand, if a bijection $\mathcal{M}_{\boldsymbol{u}}$ as in (2) exists, then the difference between the number of elements in $A_{\boldsymbol{u}}$ and $B_{\boldsymbol{u}}$ that are inside any $n$-cube $Q$ is trivially at most $(2 M+1)^{d} \cdot 2 d \cdot n^{d-1}=O\left(n^{d-1}\right)$. Theorems 1.1 and 1.5 in [17] discuss to which degree the above conditions are best possible.

In this paper we establish the following sufficient condition for measurable equivalence. 
TheORem 2.2. Let $k \geqslant 1$ and $d \geqslant 2$ be integers, let $\delta>0$, let $\boldsymbol{x}_{1}, \ldots, \boldsymbol{x}_{d} \in$ $\mathbb{T}^{k}$ be free, and let a function $\Psi:\left\{2^{i}: i \in \mathbb{N}\right\} \rightarrow \mathbb{R}$ satisfy

$$
\sum_{i=0}^{\infty} \frac{\Psi\left(2^{i}\right)}{2^{(d-2) i}}<\infty .
$$

Define $\Phi:\left\{2^{i}: i \in \mathbb{N}\right\} \rightarrow \mathbb{R}$ by $\Phi\left(2^{i}\right):=2^{i} \cdot \Psi\left(2^{i}\right)$ for $i \in \mathbb{N}$.

(1) If Lebesgue measurable sets $A, B \subseteq \mathbb{T}^{k}$ are $\Psi$-uniform of density $\delta$ with respect to every $(d-1)$-tuple of distinct vectors from $\left\{\boldsymbol{x}_{1}, \ldots, \boldsymbol{x}_{d}\right\}$, then $A \stackrel{\text { Tr }}{\sim} B$, where all pieces are Lebesgue measurable and are translated by integer combinations of the vectors $\boldsymbol{x}_{j}$.

(2) If Baire sets $A, B \subseteq \mathbb{T}^{k}$ are $\Phi$-uniform of density $\delta$ with respect to $\boldsymbol{x}_{1}, \ldots, \boldsymbol{x}_{d}$, then $A \stackrel{\text { Tr }}{\sim} B$, where all pieces are Baire and are translated by integer combinations of the vectors $\boldsymbol{x}_{j}$.

Remark 2.3. In the notation of Theorem 2.2, if $X \subseteq \mathbb{T}^{k}$ is $\Psi$-uniform with respect to any $d-1$ vectors from $\left\{\boldsymbol{x}_{1}, \ldots, \boldsymbol{x}_{d}\right\}$, then $X$ is $\Phi$-uniform with respect to $\boldsymbol{x}_{1}, \ldots, \boldsymbol{x}_{d}$. (Indeed, we can trivially represent any $d$-dimensional $2^{i}$ cube in $\mathbb{Z}^{d}$ as the disjoint union of $2^{i}$ copies of the $(d-1)$-dimensional $2^{i}$-cube.) Thus the uniformity assumption of Part 1 is stronger than that of Part 2 (or of Theorem 2.1). We do not know if the $\Phi$-uniformity alone is sufficient in Part 1.

The following result of Laczkovich [16] shows how to pick vectors that satisfy Theorem 2.1. Since it is not explicitly stated in [16], we briefly sketch its proof.

Lemma 2.4 (Laczkovich [16]). Let an integer $k \geqslant 1$ and a set $X \subseteq \mathbb{T}^{k}$ satisfy $\operatorname{dim}_{\square}(\partial X)<k$. Then there is $d(X)$ such that, for every $d \geqslant d(X)$, if we select uniformly distributed independent random vectors $\boldsymbol{x}_{1}, \ldots, \boldsymbol{x}_{d} \in \mathbb{T}^{k}$ then with probability 1 there is $C=C\left(X ; \boldsymbol{x}_{1}, \ldots, \boldsymbol{x}_{d}\right)$ such that $X$ is $\Phi$-uniform of density $\lambda(X)$ with respect to $\boldsymbol{x}_{1}, \ldots, \boldsymbol{x}_{d}$, where $\Phi\left(2^{i}\right):=C \cdot 2^{(d-2) i}$ for $i \in \mathbb{N}$.

Sketch of Proof. By a box in $\mathbb{T}^{k}$ we mean a product of $k$ sub-intervals of $[0,1)$. Let $d \geqslant 1$ be arbitrary and let $\boldsymbol{x}_{1}, \ldots, \boldsymbol{x}_{d} \in \mathbb{T}^{k}$ be random. By applying the Erdős-Turán-Koksma inequality, one can show that, with probability 1, there is $C^{\prime}=C^{\prime}\left(\boldsymbol{x}_{1}, \ldots, \boldsymbol{x}_{d}\right)$ such that, for every box $Y \subseteq \mathbb{T}^{k}, \boldsymbol{u} \in \mathbb{T}^{k}$, and $N$-cube $Q \subseteq \mathbb{Z}^{d}$, we have that

$$
|| Y_{\boldsymbol{u}} \cap Q|-\lambda(Y)| Q|| \leqslant \Upsilon(N):=C^{\prime} \log ^{k+d+1} N,
$$

see [16, Lemma 2]. In other words, boxes have very small discrepancy with respect to arbitrary cubes. (In particular, each box is $\Upsilon$-uniform.)

So, assume that (6) holds and that $\boldsymbol{x}_{1}, \ldots, \boldsymbol{x}_{d}$ are free. Fix a real $\alpha \in(0,1]$ satisfying $\operatorname{dim}_{\square}(\partial X)<k-\alpha$. A result of Niederreiter and Wills [28, Kollorar 4] implies that the set $X$ is $\Psi$-uniform (with respect to $\boldsymbol{x}_{1}, \ldots, \boldsymbol{x}_{d}$ ) for some $\Psi(N)$ 

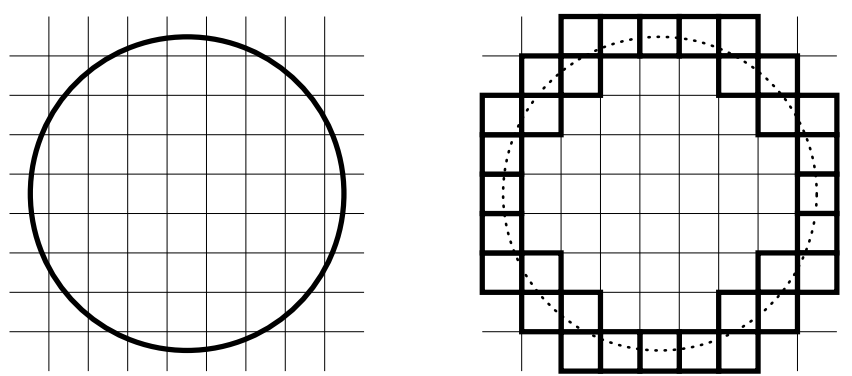

Figure 1. i) Circle $\partial X$ and $\varepsilon$-regular grid; iii) boxes in $\mathcal{I}$

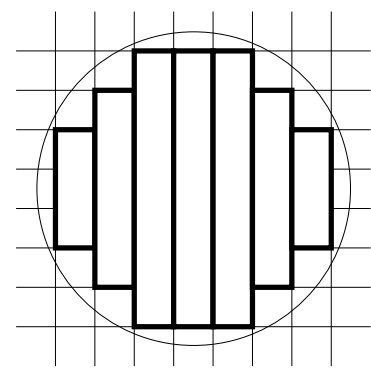

that grows as $O\left(\Upsilon(N)^{\alpha / k} N^{d-\alpha d / k}\right)$ as $N \rightarrow \infty$. In particular, we can satisfy Lemma 2.4 by letting $d(X)$ be any integer such that $\alpha d(X) / k>2$. We refer the reader to $[16$, Page 62$]$ for further details.

Let us also outline the ideas behind [28, Kollorar 4] in order to show how the box dimension of $\partial X$ comes into play. The definition of $\alpha$ implies that the measure of points within $L^{\infty}$-distance $\varepsilon$ from the boundary of $X$ is at most $\varepsilon^{\alpha}$ for all small $\varepsilon>0$. Let $N$ be large and let $\varepsilon:=\left\lfloor\left(N^{d} / \Upsilon(N)\right)^{1 / k}\right\rfloor^{-1}$. Partition $\mathbb{T}^{k}$ into a grid of boxes which is $\varepsilon$-regular, meaning that side lengths are all equal to $\varepsilon$. Let $\mathcal{B}$ consist of those boxes that intersect $\partial X$. By the definition of $\alpha$, we have that $|\mathcal{B}| \leqslant \varepsilon^{\alpha} / \varepsilon^{k}$. Next, iteratively merge any two boxes in the interior of $X$ if they have the same projection on the first $k-1$ coordinates and share a $(k-1)$-dimensional face. Let $\mathcal{I}$ be the set of the final boxes in the interior of $X$. Figure 1 illustrates the special case when $X$ is a disk. The size of $\mathcal{I}$ is at most $\varepsilon^{-k+1}$ (the number of possible projections) plus $|\mathcal{B}|$ (as each box in $\mathcal{B}$ can "prevent" at most one merging).

Pick any $\boldsymbol{u} \in \mathbb{T}^{k}$ and an $N$-cube $Q \subseteq \mathbb{Z}^{d}$. We take the dual point of view where we fix $Q^{\prime}:=\left\{\boldsymbol{u}+\sum_{j=1}^{d} n_{j} \boldsymbol{x}_{j}: \boldsymbol{n} \in Q\right\} \subseteq \mathbb{T}^{k}$ and measure its discrepancy with respect to boxes. Namely, we have by $(6)$ that $D^{\prime}(Y) \leqslant \Upsilon(N)$ for every box $Y \subseteq \mathbb{T}^{k}$, where we define $D^{\prime}(Y):=|| Q^{\prime} \cap Y\left|-\lambda(Y) N^{d}\right|$. This implies that

$$
D^{\prime}(X) \leqslant \sum_{Y \in \mathcal{I}} D^{\prime}(Y)+\sum_{Y \in \mathcal{B}} D^{\prime}(Y \cap X) \leqslant|\mathcal{I}| \cdot \Upsilon(N)+|\mathcal{B}|\left(\varepsilon^{k} N^{d}+\Upsilon(N)\right),
$$

giving the stated upper bound after routine simplifications.

Thus Lemma 2.4 shows that the uniformity assumption of Part 2 of Theorem 2.2 can be satisfied if the sets $A$ are $B$ are as in Theorem 1.2. The lemma also suffices for Part 1 of Theorem 2.2, thus leading to the proof of Theorem 1.2 as follows. 
Proof of Theorem 1.2. Observe that the assumption $\operatorname{dim}_{\square}(\partial X)<k$ implies that $X \subseteq \mathbb{T}^{k}$ is both Baire and Lebesgue measurable. For example, let us argue that $X$ is Baire. Every set is the union of its interior (an open set) and a subset of its boundary. So it is enough to show that $\partial X$ is nowhere dense. Take any ball $U \subseteq \mathbb{T}^{k}$ of radius $r>0$. As $\varepsilon \rightarrow 0$, the $\varepsilon$-neighbourhood of $\partial X$ has measure at most $\varepsilon^{\alpha}$ for some constant $\alpha>0$. This is strictly smaller than $((r-\varepsilon) / r)^{k} \lambda(U)$, the volume of the ball $U^{\prime}$ concentric to $U$ of radius $r-\varepsilon$, so at least one point $\boldsymbol{x} \in U^{\prime}$ is uncovered. The open ball of radius $\varepsilon$ around $\boldsymbol{x}$ lies entirely inside $U$ and avoids $\partial X$. Thus $\partial X$ is nowhere dense, as desired.

Therefore, the sets $A$ and $B$ in Theorem 1.2 are both Baire and Lebesgue measurable. Next, let us show that we can satisfy the uniformity assumption of Part 1 of Theorem 2.2.

Let $d:=\max (d(A), d(B))+1$, where $d(X)$ is the function provided by Lemma 2.4. Fix free vectors $\boldsymbol{x}_{1}, \ldots, \boldsymbol{x}_{d} \in \mathbb{T}^{k}$ such that every $(d-1)$-tuple of them satisfies the conclusion of Lemma 2.4 for both $A$ and $B$. Such vectors exist since the desired properties hold with probability 1 if we sample the vectors $\boldsymbol{x}_{j}$ independently. Let $C<\infty$ be the maximum, over all choices of $X \in\{A, B\}$ and integers $1 \leqslant i_{1}<\ldots<i_{d-1} \leqslant d$, of the corresponding constants $C\left(X ; \boldsymbol{x}_{i_{1}}, \ldots, \boldsymbol{x}_{i_{d-1}}\right)$. Then the assumptions of Part 1 of Theorem 2.2 hold with $\Psi\left(2^{i}\right):=C \cdot 2^{(d-3) i}$ (and the same function works with Part 2).

Theorem 2.2 implies that $A$ and $B$ are equivalent with Baire (resp. Lebesgue) measurable pieces. Allowing empty pieces, let this be witnessed respectively by partitions $A=\cup_{\boldsymbol{v} \in \mathcal{V}} A_{\boldsymbol{v}}^{\prime}$ and $A=\cup_{\boldsymbol{v} \in \mathcal{V}} A_{\boldsymbol{v}}^{\prime \prime}$ for some finite $\mathcal{V} \subseteq \mathbb{T}^{k}$, where the pieces $A_{\boldsymbol{v}}^{\prime}$ and $A_{\boldsymbol{v}}^{\prime \prime}$ are translated by $\boldsymbol{v}$. These equidecompositions can be "merged" as follows. Take a nullset $X \subseteq \mathbb{T}^{k}$ such that $\mathbb{T}^{k} \backslash X$ is meagre; the existence of $X$ follows from e.g. [29, Theorem 1.6]. We can additionally assume that $X$ is invariant under all translations from $\mathcal{V}$. (For example, take the union of all translates of $X$ by integer combinations of the vectors from $\mathcal{V}$; it is still a nullset since we take countably many translates.) Now, we combine the Baire partition of $A$ restricted to $X$ with the Lebesgue partition restricted to $\mathbb{T}^{k} \backslash X$. Specifically, let $A_{\boldsymbol{v}}:=\left(A_{\boldsymbol{v}}^{\prime} \cap X\right) \cup\left(A_{\boldsymbol{v}}^{\prime \prime} \backslash X\right)$ for $\boldsymbol{v} \in \mathcal{V}$. Clearly, these sets partition $A$ while, by the invariance of $X$, the corresponding translates $A_{\boldsymbol{v}}+\boldsymbol{v}$, for $\boldsymbol{v} \in \mathcal{V}$, partition $B$. Also, each part $A_{\boldsymbol{v}}$ is both Baire and Lebesgue measurable. This proves Theorem 1.2.

\section{Some common definitions and results}

Our proofs of Parts 1 and 2 of Theorem 2.2 proceed somewhat differently. This section collects some definitions and auxiliary results that are common to both parts. Here, let measurable mean Baire or Lebesgue measurable, depending on which $\sigma$-algebra we are interested in. 
Since we will study equidecompositions from graph-theoretic point of view, we find it convenient to adopt some notions of graph theory to our purposes as follows.

By a bipartite graph we mean a triple $G=\left(V_{1}, V_{2}, E\right)$, where $V_{1}$ and $V_{2}$ are (finite or infinite) vertex sets and $E \subseteq V_{1} \times V_{2}$ is a set of edges. (Note that $E$ consists of ordered pairs to avoid ambiguities when $V_{1}$ and $V_{2}$ are not disjoint.) The subgraph induced by sets $X_{1}$ and $X_{2}$ is

$$
G\left[X_{1}, X_{2}\right]:=\left(V_{1} \cap X_{1}, V_{2} \cap X_{2}, E \cap\left(X_{1} \times X_{2}\right)\right) .
$$

A matching in $G$ is a subset $\mathcal{M}$ of $E$ which gives a partial injection from $V_{1}$ to $V_{2}$ (that is, if $(a, b)$ and $\left(a^{\prime}, b^{\prime}\right)$ are distinct pairs in $\mathcal{M}$ then $a \neq a^{\prime}$ and $\left.b \neq b^{\prime}\right)$. In fact, we will identify a matching with the corresponding partial injection. In particular, the sets of matched points in $V_{1}$ and $V_{2}$ can be respectively denoted by $\mathcal{M}^{-1}\left(V_{2}\right)$ and $\mathcal{M}\left(V_{1}\right)$. A matching $\mathcal{M}$ is perfect if it is a bijection from $V_{1}$ to $V_{2}$ (that is, if $\mathcal{M}\left(V_{1}\right)=V_{2}$ and $\mathcal{M}^{-1}\left(V_{2}\right)=V_{1}$ ). For a set $X$ lying in one part of $G$, let its neighbourhood $\Gamma(X)$ consist of those vertices in the other part that are connected by at least one edge to $X$. (In the functional notation, we have $\Gamma(X)=E(X)$ for $X \subseteq A$ and $\Gamma(X)=E^{-1}(X)$ for $X \subseteq B$.) If $G$ is locally finite (that is, every degree $|\Gamma(\{x\})|$ is finite), then Rado's theorem [30] states that $G$ has a perfect matching if and only if

$$
|\Gamma(X)| \geqslant|X|, \quad \text { for every finite subset } X \text { of } A \text { or } B .
$$

Note that if $V_{1} \cap V_{2}=\emptyset$ then we get the standard notions of graph theory with respect to the corresponding undirected graph on $V_{1} \cup V_{2}$.

Thus, an equidecomposition between $A, B \subseteq \mathbb{T}^{k}$ where all translations are restricted to the set $\mathcal{V}_{M}$ that was defined in (1) is nothing else than a perfect matching in the bipartite graph

$$
\mathcal{G}:=(A, B, E),
$$

where $E$ consists of all pairs $(\boldsymbol{a}, \boldsymbol{b}) \in A \times B$ with $\boldsymbol{b}-\boldsymbol{a} \in \mathcal{V}_{M}$.

Assume from now on that both $A$ and $B$ are measurable (which will be the case in all applications). Then, each of the vertex parts of the graph $\mathcal{G}$ is additionally endowed with the $\sigma$-algebra of measurable sets; objects of this type appear in orbit equivalence [13], limits of sparse graphs [24], and other areas. A matching $\mathcal{M}$ in $\mathcal{G}$ is called measurable if the set $\{\boldsymbol{a} \in A: \mathcal{M}(\boldsymbol{a})-\boldsymbol{a}=\boldsymbol{v}\}$ is measurable for each $\boldsymbol{v} \in \mathcal{V}_{M}$.

Also, we will consider the subgraphs of $\mathcal{G}$ induced by cosets, viewing these as graphs on subsets of $\mathbb{Z}^{d}$. Namely, for $\boldsymbol{u} \in \mathbb{T}^{k}$, consider the bipartite graph $\mathcal{G}_{\boldsymbol{u}}:=\left(A_{\boldsymbol{u}}, B_{\boldsymbol{u}}, E_{\boldsymbol{u}}\right)$, where

$$
E_{\boldsymbol{u}}:=\left\{(\boldsymbol{a}, \boldsymbol{b}) \in A_{\boldsymbol{u}} \times B_{\boldsymbol{u}}:\|\boldsymbol{a}-\boldsymbol{b}\|_{\infty} \leqslant M\right\} .
$$


Again, a bijection $\mathcal{M}_{\boldsymbol{u}}: A_{\boldsymbol{u}} \rightarrow B_{\boldsymbol{u}}$ as in (2) is nothing else than a perfect matching in $\mathcal{G}_{\boldsymbol{u}}$ and, in order to prove Theorem 2.1, it is enough to show that each $\mathcal{G}_{\boldsymbol{u}}$ has at least one perfect matching. For the proof of Theorem 2.2, we will also need that the dependence on $\boldsymbol{u}$ is "equivariant" and "measurable" in the following sense.

Namely, we call the family $\left(\mathcal{M}_{\boldsymbol{u}}\right)_{\boldsymbol{u} \in \mathbb{T}^{k}}$ with $\mathcal{M}_{\boldsymbol{u}}$ being a matching in $\mathcal{G}_{\boldsymbol{u}}$ equivariant if, for all $\boldsymbol{u} \in \mathbb{T}^{k}$ and $\boldsymbol{n} \in \mathbb{Z}^{d}$, we have

$$
\mathcal{M}_{\boldsymbol{u}+n_{1} \boldsymbol{x}_{1}+\ldots+n_{d} \boldsymbol{x}_{d}}=\left\{(\boldsymbol{a}-\boldsymbol{n}, \boldsymbol{b}-\boldsymbol{n}):(\boldsymbol{a}, \boldsymbol{b}) \in \mathcal{M}_{\boldsymbol{u}}\right\} .
$$

Note that, if (10) holds, then we can define a partial injection $\mathcal{M}: A \rightarrow B$ as follows. In order to find the image $\mathcal{M}(\boldsymbol{a})$ of $\boldsymbol{a} \in A$, take any $\boldsymbol{u}$ such that $\boldsymbol{a}$ is in the coset of $\boldsymbol{u}$, say $\boldsymbol{a}=\boldsymbol{u}+\sum_{j=1}^{d} n_{j} \boldsymbol{x}_{j}$ with $\boldsymbol{n} \in \mathbb{Z}^{d}$. Note that $\boldsymbol{n} \in A_{\boldsymbol{u}}$. If $\boldsymbol{n}$ is not matched by $\mathcal{M}_{\boldsymbol{u}}$, then let $\mathcal{M}(\boldsymbol{a})$ be undefined; otherwise let $\mathcal{M}(\boldsymbol{a}):=\boldsymbol{u}+\sum_{j=1}^{d} m_{j} \boldsymbol{x}_{j} \in B$, where $\boldsymbol{m}:=\mathcal{M}_{\boldsymbol{u}}(\boldsymbol{n})$. It is easy to see that, by (10), the definition of $\mathcal{M}(\boldsymbol{a})$ does not depend on the choice of $\boldsymbol{u}$ (and it will often be notationally convenient to take $\boldsymbol{u}=\boldsymbol{a}$ ).

The concept of equivariance can be applied to other kinds of objects, with the definition being the obvious adaptation of (10) in all cases that we will encounter. Namely, the "meta-definition" is that if we shift the coset reference point from $\boldsymbol{u}$ to $\boldsymbol{u}+n_{1} \boldsymbol{x}_{1}+\ldots+n_{d} \boldsymbol{x}_{d}$ for some $\boldsymbol{n} \in \mathbb{Z}^{d}$, then the object does not change, i.e. its new coordinates are all shifted by $\boldsymbol{- n}$. For example, for every $X \subseteq \mathbb{T}^{k}$ the family of sets $\left(X_{\boldsymbol{u}}\right)_{\boldsymbol{u} \in \mathbb{T}^{k}}$ is equivariant and, conversely, every equivariant family of subsets of $\mathbb{Z}^{d}$ gives a subset of $\mathbb{T}^{k}$. As another example, the family $\left(\mathcal{G}_{\boldsymbol{u}}\right)_{\boldsymbol{u} \in \mathbb{T}^{k}}$ is equivariant and corresponds to the bipartite graph $\mathcal{G}$ defined in (9).

We call an equivariant family $\left(\mathcal{M}_{\boldsymbol{u}}\right)_{\boldsymbol{u} \in \mathbb{T}^{k}}$ with $\mathcal{M}_{\boldsymbol{u}}$ being a (not necessarily perfect) matching in $\mathcal{G}_{\boldsymbol{u}}$ measurable if the natural encoding of the corresponding matching $\mathcal{M}$ by a function $\mathbb{T}^{k} \rightarrow[-M, M]^{d} \cup\{$ UNMATCHED $\}$ is measurable. (Note that this is equivalent to the measurability of $\mathcal{M}$ as defined after (9).) Again, this concept can be applied to other objects: for example, an equivariant family $\left(X_{\boldsymbol{u}}\right)_{\boldsymbol{u} \in \mathbb{T}^{k}}$ of subsets of $\mathbb{Z}^{d}$ is called measurable if the corresponding encoding $\mathbb{T}^{k} \rightarrow\{0,1\}$ (i.e. the corresponding set $X \subseteq \mathbb{T}^{k}$ ) is measurable.

Thus, if we can find an equivariant and measurable family $\left(\mathcal{M}_{\boldsymbol{u}}\right)_{\boldsymbol{u} \in \mathbb{T}^{k}}$ with $\mathcal{M}_{\boldsymbol{u}}$ being a perfect matching in $\mathcal{G}_{\boldsymbol{u}}$ for each $\boldsymbol{u} \in \mathbb{T}^{k}$, then we have a measurable bijection $\mathcal{M}: A \rightarrow B$. Furthermore, the differences $\mathcal{M}(\boldsymbol{a})-\boldsymbol{a}$ for $\boldsymbol{a} \in A$ are all restricted to the finite set $\mathcal{V}_{M}$, giving the required measurable equivalence $A \stackrel{\operatorname{Tr}}{\sim} B$.

Thus, informally speaking, each element $\boldsymbol{n} \in A_{\boldsymbol{u}}$ has to find its match $\mathcal{M}_{\boldsymbol{u}}(\boldsymbol{n})$ in a measurable way which is also invariant under shifting the whole coset by any integer vector. For example, a measurable inclusion-maximal matching $\mathcal{M}$ between $A$ and $B$ can be constructed by iteratively applying the 
following over all $\boldsymbol{v} \in \mathcal{V}_{M}$ : add to the current matching $\mathcal{M}$ all possible pairs $(\boldsymbol{a}, \boldsymbol{a}+\boldsymbol{v})$, i.e. for all $\boldsymbol{a}$ in the set

$$
X:=\left(A \backslash \mathcal{M}^{-1}(B)\right) \cap((B \backslash \mathcal{M}(A))-\boldsymbol{v}) .
$$

Clearly, $X$ is measurable if $\mathcal{M}$ is; thus one iteration preserves the measurability of $\mathcal{M}$. Also, each of the above iterations can be determined by a "local" rule within a coset: namely, the match of a vertex $\boldsymbol{n} \in A_{\boldsymbol{u}}$ depends only on the current picture inside the ball of radius $M$ around $\boldsymbol{n}$ (while the new values of $\mathcal{M}$ can be determined in parallel).

Let us formalise the above idea. For $r \in \mathbb{N}$, a radius-r local rule (or simply an $r$-local rule) is a function $\mathcal{R}: \mathbb{N}^{Q_{r}} \rightarrow \mathbb{N}$, where $Q_{r}:=[-r, r]^{d} \subseteq \mathbb{Z}^{d}$; it instructs how to transform any function $g: \mathbb{T}^{k} \rightarrow \mathbb{N}$ into another function $g^{\mathcal{R}}: \mathbb{T}^{k} \rightarrow \mathbb{N}$. Namely, for $\boldsymbol{u} \in \mathbb{T}^{k}$, we define

$$
g^{\mathcal{R}}(\boldsymbol{u}):=\mathcal{R}\left(\left.g_{\boldsymbol{u}}\right|_{Q_{r}}\right),
$$

where $g_{\boldsymbol{u}}: \mathbb{Z}^{d} \rightarrow \mathbb{N}$ is the coset version of $g$ (i.e. $g_{\boldsymbol{u}}(\boldsymbol{n}):=g\left(\boldsymbol{u}+\sum_{j=1}^{d} n_{j} \boldsymbol{x}_{j}\right)$ for $\left.\boldsymbol{n} \in \mathbb{Z}^{d}\right)$ and $\left.g_{\boldsymbol{u}}\right|_{Q_{r}}: Q_{r} \rightarrow \mathbb{N}$ denotes its restriction to the cube $Q_{r}$.

Lemma 3.1. If $g: \mathbb{T}^{k} \rightarrow \mathbb{N}$ is a measurable function, then, for any $r$-local rule $\mathcal{R}$, the function $g^{\mathcal{R}}: \mathbb{T}^{k} \rightarrow \mathbb{N}$ is measurable.

Proof. For any function $f: Q_{r} \rightarrow \mathbb{N}$ define

$$
X_{f}:=\left\{\boldsymbol{u} \in \mathbb{T}^{k}:\left.g_{\boldsymbol{u}}\right|_{Q_{r}}=f\right\} .
$$

Thus $\boldsymbol{u} \in X_{f}$ if and only if $g\left(\boldsymbol{u}+\sum_{j=1}^{d} n_{j} \boldsymbol{x}_{j}\right)=f(\boldsymbol{n})$ for every $\boldsymbol{n} \in Q_{r}$. This means that $X_{f}$ is the intersection, over $\boldsymbol{n} \in Q_{r}$, of the translates of $g^{-1}(f(\boldsymbol{n})) \subseteq \mathbb{T}^{k}$ by the vector $-\sum_{j=1}^{d} n_{j} \boldsymbol{x}_{j}$. Each of these translates is a measurable set by the measurability of $g: \mathbb{T}^{k} \rightarrow \mathbb{N}$.

Furthermore, the pre-image of any $i \in \mathbb{N}$ under $g^{\mathcal{R}}$ is the disjoint union of $X_{f}$ over $f$ with $\mathcal{R}(f)=i$. This union is measurable as there are only countably many possible functions $f$.

To avoid confusion when we have different graphs on $\mathbb{Z}^{d}$, the distance between $\boldsymbol{x}, \boldsymbol{y} \in \mathbb{Z}^{d}$ will always mean the $L^{\infty}$-distance between vectors:

$$
\operatorname{dist}(\boldsymbol{x}, \boldsymbol{y})=\|\boldsymbol{x}-\boldsymbol{y}\|_{\infty} .
$$

Also, we use the standard definition of the distance between sets:

$$
\operatorname{dist}(X, Y):=\min \{\operatorname{dist}(\boldsymbol{x}, \boldsymbol{y}): \boldsymbol{x} \in X, \boldsymbol{y} \in Y\}, \quad X, Y \subseteq \mathbb{Z}^{d} .
$$

For $X \subseteq \mathbb{Z}^{k}$ and $m \in \mathbb{N}$, we define the $m$-ball around $X$ to be

$$
\operatorname{dist}_{\leqslant m}(X):=\left\{\boldsymbol{n} \in \mathbb{Z}^{d}: \operatorname{dist}(\boldsymbol{n}, X) \leqslant m\right\} .
$$


A collection $\mathcal{X}$ of elements or subsets of $\mathbb{Z}^{d}$ is $r$-sparse if the distance between any two distinct members of $\mathcal{X}$ is strictly larger than $r$. A set $X \subseteq \mathbb{T}^{k}$ is called $r$-sparse if $X_{\boldsymbol{u}} \subseteq \mathbb{Z}^{d}$ is $r$-sparse for each $\boldsymbol{u} \in \mathbb{T}^{k}$.

Lemma 3.2. For every $r$ there is a Borel measurable map $\chi: \mathbb{T}^{k} \rightarrow[t]$ for some $t \in \mathbb{N}$ such that each pre-image $\chi^{-1}(i) \subseteq \mathbb{T}^{k}, i \in[t]$, is r-sparse.

Proof. The existence of $\chi$ follows from the more general results of Kechris, Solecki and Todorcevic [14].

Alternatively, pick $n \in \mathbb{N}$ such that $1 / n$ is smaller than the minimum distance inside the finite set $\left\{\sum_{j=1}^{d} n_{j} \boldsymbol{x}_{j}: \boldsymbol{n} \in \mathbb{Z}^{d},\|\boldsymbol{n}\|_{\infty} \leqslant r\right\} \subseteq \mathbb{T}^{k}$. Then any subset of $\mathbb{T}^{k}$ of diameter at most $1 / n$ is $r$-sparse. Thus we can take for $\chi$ any function that has the half-open boxes of the $(1 / n)$-regular grid on $\mathbb{T}^{k}$ as its pre-images (where $t=n^{k}$ ).

For $X \subseteq \mathbb{Z}^{d}$, its boundary $\partial X$ is the set of ordered pairs $(\boldsymbol{m}, \boldsymbol{n})$ such that $\boldsymbol{m} \in X, \boldsymbol{n} \in \mathbb{Z}^{d} \backslash X$ and the vector $\boldsymbol{n}-\boldsymbol{m}$ has zero entries except one entry equal to \pm 1 (i.e. $\boldsymbol{n}-\boldsymbol{m}= \pm \boldsymbol{e}_{j}$ for a standard basis vector $\boldsymbol{e}_{j}$ ). The perimeter of $X$ is $p(X):=|\partial X|$. In other words, the perimeter of $X$ is the number of edges leaving $X$ in the standard $2 d$-regular graph on $\mathbb{Z}^{d}$.

We will need a lower bound on the perimeter of a finite set $X \subseteq \mathbb{Z}^{d}$ in terms of its size. While the exact solution to this edge-isoperimetric problem is known (see Ahlswede and Bezrukov [1, Theorem 2]), we find it more convenient to use the old result of Loomis and Whitney [23] that gives a bound which is easy to state and suffices for our purposes.

Lemma 3.3. For every finite $X \subseteq \mathbb{Z}^{d}$ we have $p(X) \geqslant 2 d \cdot|X|^{(d-1) / d}$.

Proof. A result of Loomis and Whitney [23, Theorem 2] directly implies that $|X|^{d-1} \leqslant \prod_{j=1}^{d}\left|X_{j}\right|$, where $X_{1}, \ldots, X_{d} \subseteq \mathbb{Z}^{d-1}$ are all $(d-1)$-dimensional projections of $X$. Thus, by the Geometric-Arithmetic Mean Inequality, we obtain the required:

$$
p(X) \geqslant 2 \sum_{j=1}^{d}\left|X_{j}\right| \geqslant 2 d\left(\prod_{j=1}^{d}\left|X_{j}\right|\right)^{1 / d} \geqslant 2 d \cdot|X|^{(d-1) / d} .
$$

\section{Proof of Part 1 of Theorem 2.2}

Throughout this section, measurable means Lebesgue measurable.

4.1. Overview of main ideas and steps. First, let us define some global constants that will be used for proving Part 1 of Theorem 2.2. Recall that we are given the measurable sets $A, B \subseteq \mathbb{T}^{k}$ that are $\Psi$-uniform of density 
$\delta>0$ with respect to any $d-1$ of the vectors $\boldsymbol{x}_{1}, \ldots, \boldsymbol{x}_{d} \in \mathbb{T}^{k}$. As we mentioned in Remark 2.3, this implies that $A$ and $B$ are $\Phi$-uniform with respect to $\boldsymbol{x}_{1}, \ldots, \boldsymbol{x}_{d} \in \mathbb{T}^{k}$. (Recall that $\Phi\left(2^{i}\right):=2^{i} \cdot \Psi\left(2^{i}\right)$ for $i \in \mathbb{N}$.) It easily follows (e.g. from Lemma 4.1 below) that $\lambda(A)=\lambda(B)=\delta$.

Given $A, B, \Psi, \boldsymbol{x}_{1}, \ldots, \boldsymbol{x}_{d}$, choose a large constant $M$ (namely, it has to satisfy Lemma 4.2 below). Let $\left(N_{i}\right)_{i \in \mathbb{N}}$ be a strictly increasing sequence, consisting of integer powers of 2 such that $\sum_{i=0}^{\infty} N_{i}^{2} / N_{i+1}<\infty$. When some index $i$ goes to infinity, we may use asymptotic notation, such as $O(1)$, to denote constants that do not depend on $i$.

We will be constructing the desired measurable perfect matching in the bipartite graph $\mathcal{G}=(A, B, E)$ that was defined by (9) by iteratively improving partial matchings. Namely, each Iteration $i$ replaces the previous partial measurable matching $\mathcal{M}_{i-1}$ by a "better" matching $\mathcal{M}_{i}$ using finitely many local rules. Clearly, the new family $\left(\mathcal{M}_{i, \boldsymbol{u}}\right)_{\boldsymbol{u} \in \mathbb{T}^{k}}$ is still equivariant and, by Lemma 3.1, measurable. We wish to find matchings $\left(\mathcal{M}_{i}\right)_{i \in \mathbb{N}}$ such that for a.e. (almost every) $\boldsymbol{a} \in A$ the sequence $\mathcal{M}_{i}(\boldsymbol{a})$ stabilises eventually, that is, there are $n \in \mathbb{N}$ and $\boldsymbol{b} \in B$ such that $\mathcal{M}_{i}(\boldsymbol{a})=\boldsymbol{b}$ for all $i \geqslant n$. In this case, we agree that the final partial map $\mathcal{M}$ maps $\boldsymbol{a}$ to $\boldsymbol{b}$. Equivalently,

$$
\mathcal{M}:=\cup_{i \in \mathbb{N}} \cap_{j=i}^{\infty} \mathcal{M}_{j}
$$

where we view matchings in $\mathcal{G}=(A, B, E)$ as subsets of $E$. Clearly, any family $\left(\mathcal{M}_{\boldsymbol{u}}\right)_{\boldsymbol{u} \in \mathbb{T}^{k}}$ of matchings obtained this way is equivariant and measurable.

In order to guarantee that almost every vertex of $A$ is matched (i.e. that $\left.\lambda\left(\mathcal{M}^{-1}(B) \backslash A\right)=0\right)$, it is enough to establish the following two properties:

$$
\begin{aligned}
\lim _{i \rightarrow \infty} \lambda\left(\mathcal{M}_{i}^{-1}(B)\right) & =\lambda(A), \\
\sum_{i=0}^{\infty} \lambda\left(\left(\mathcal{M}_{i} \triangle \mathcal{M}_{i+1}\right)^{-1}(B)\right) & <\infty,
\end{aligned}
$$

where $\mathcal{M}_{i} \triangle \mathcal{M}_{i+1} \subseteq E$ is the symmetric difference of $\mathcal{M}_{i}$ and $\mathcal{M}_{i+1}$, and thus $\left(\mathcal{M}_{i} \triangle \mathcal{M}_{i+1}\right)^{-1}(B)$ is the set of those $\boldsymbol{a} \in A$ such that $\mathcal{M}_{i}(\boldsymbol{a}) \neq \mathcal{M}_{i+1}(\boldsymbol{a})$, including the cases when only one of these is defined.

Indeed, suppose that (13) and (14) hold. Let $A_{i}^{\prime}$ consist of those vertices of $A$ whose match is modified at least once after Iteration $i$, that is, $A_{i}^{\prime}:=$ $\cup_{j=i}^{\infty}\left(\mathcal{M}_{j} \triangle \mathcal{M}_{j+1}\right)^{-1}(B)$. The measure $\lambda\left(A_{i}^{\prime}\right)$ tends to 0 as $i \rightarrow \infty$ because it is trivially bounded by the corresponding tail of the sum in (14). Thus the set $A^{\prime}:=\cap_{i=0}^{\infty} A_{i}^{\prime}$ of vertices in $A$ that do not stabilise eventually has measure zero. Also, for every $i \in \mathbb{N}$ we have that $\mathcal{M}^{-1}(B) \supseteq \mathcal{M}_{i}^{-1}(B) \backslash A_{i}^{\prime}$. If we consider the measure of these sets and use $(13)$, we conclude that $\lambda\left(\mathcal{M}^{-1}(B)\right) \geqslant \lambda(A)$, giving the required conclusion.

Thus, if we are successful in establishing (13) and (14), this gives an a.e. defined measurable map $\mathcal{M}$, which shows that $A \backslash A^{\prime}$ and $B \backslash B^{\prime}$ are measurably 
equivalent, for some nullsets $A^{\prime} \subseteq A$ and $B^{\prime} \subseteq B$. It is not hard to modify $\mathcal{M}$ to get rid of the exceptional sets. Namely, let $X \subseteq \mathbb{T}^{k}$ be the union of all cosets that intersect $A^{\prime} \cup B^{\prime}$. Note that $X$ is a nullset. Let $\mathcal{M}^{\prime}: A \rightarrow B$ be given by Theorem 2.1 using the same vectors $\boldsymbol{x}_{1}, \ldots, \boldsymbol{x}_{d}$. Then, $\mathcal{M}$ (resp. $\mathcal{M}^{\prime}$ ) induces a bijection $A \backslash X \rightarrow B \backslash X$ (resp. $A \cap X \rightarrow B \cap X$ ) and we can use $\mathcal{M}$ on $A \backslash X$ and $\mathcal{M}^{\prime}$ on $A \cap X$. The obtained bijection $A \rightarrow B$ is measurable since $\mathcal{M}^{\prime}$ is applied only inside the nullset $X$.

The following trivial observation will be enough in all our forthcoming estimates of the measure of "bad" sets. We say that a set $X \subseteq \mathbb{T}^{k}$ (or an equivariant family $\left.\left(X_{\boldsymbol{u}}\right)_{\boldsymbol{u} \in \mathbb{T}^{k}}\right)$ has uniform density at most $c$ if there is $r \in \mathbb{N}$ such that for every $\boldsymbol{u} \in \mathbb{T}^{k}$ and for every $r$-cube $Q \subseteq \mathbb{Z}^{d}$ we have $\left|X_{\boldsymbol{u}} \cap Q\right| \leqslant c r^{d}$.

LEMMA 4.1. If a measurable set $X \subseteq \mathbb{T}^{k}$ has uniform density at most $c$, then $\lambda(X) \leqslant c$.

Proof. Let $r \in \mathbb{N}$ witness the stated uniform density. Consider $r^{d}$ translates $X+\sum_{j=1}^{d} n_{j} \boldsymbol{x}_{j}$ over $\boldsymbol{n} \in[r]^{d}$. By our assumption, every point of $\mathbb{T}^{k}$ is covered at most $\mathrm{cr}^{d}$ times. Thus the lemma follows from the finite additivity and translation invariance of the Lebesgue measure $\lambda$.

Since our construction of the matching $\mathcal{M}_{i}$ involves "improving" $\mathcal{M}_{i-1}$ along augmenting paths, let us give the corresponding general definitions now. Given a matching $\mathcal{M}$ in a bipartite graph $G=\left(V_{1}, V_{2}, E\right)$, an augmenting path is a sequence $P=\left(v_{0}, \ldots, v_{m}\right)$ of vertices such that $v_{0} \in V_{1} \backslash \mathcal{M}^{-1}\left(V_{2}\right)$, $v_{m} \in V_{2} \backslash \mathcal{M}\left(V_{1}\right),\left(v_{i}, v_{i-1}\right) \in \mathcal{M}$ for all even $i \in[m]$, and $\left(v_{i-1}, v_{i}\right) \in E \backslash \mathcal{M}$ for all odd $i \in[m]$. In other words, we start with an unmatched vertex of $V_{1}$ and alternate between edges in $E \backslash \mathcal{M}$ and $\mathcal{M}$ until we reach an unmatched vertex of $V_{2}$; note that all even (resp. odd) numbered vertices necessarily belong to the same part and are distinct. The length of $P$ is $m$, the number of edges in it; clearly, it has to be odd. If we flip the path $P$, that is, remove $\left(v_{i}, v_{i-1}\right)$ from $\mathcal{M}$ for all even $i \in[m]$ and add $\left(v_{i-1}, v_{i}\right)$ to $\mathcal{M}$ for all odd $i \in[m]$, then we obtain another matching that improves $\mathcal{M}$ by covering two extra vertices. A matching in a finite graph is maximum if it has the largest number of edges among all matchings.

As we already mentioned, we try to achieve (13) and (14) by iteratively flipping augmenting paths using some local rules. We have to be careful how we guide the paths since it is not a priori clear that if two unmatched points from different parts are close to each other in $\mathcal{G}_{\boldsymbol{u}}$, then there is a relatively short augmenting path (or any augmenting path at all).

The following lemma gives us some control over this. A rectangle $R \subseteq \mathbb{Z}^{d}$ is the product of $d$ finite intervals of integers, $R=\prod_{j=1}^{d}\left[a_{j}, b_{j}\right]$. Its side lengths 
are $b_{j}-a_{j}+1, j \in[d]$. We say that $R$ is $\rho$-balanced if the ratio of any two side lengths is at most $\rho$.

Lemma 4.2. Let the assumptions of Part 1 of Theorem 2.2 hold and let $M=M\left(A, B, \Psi, \boldsymbol{x}_{1}, \ldots, \boldsymbol{x}_{d}\right)$ be sufficiently large. Take arbitrary $\boldsymbol{u} \in \mathbb{T}^{k}$ and a 3 -balanced rectangle $R \subseteq \mathbb{Z}^{d}$. If $\mathcal{M}$ is a matching in $\mathcal{G}_{\boldsymbol{u}}[R, R]$ (the subgraph of $\mathcal{G}_{\boldsymbol{u}}$ induced by $R$, as defined in (7)) that misses at least one vertex in each part, then $\mathcal{G}_{\boldsymbol{u}}[R, R]$ contains an augmenting path whose length is at most the maximum side length of $R$. In particular, every maximum matching in $\mathcal{G}_{\boldsymbol{u}}[R, R]$ completely covers one part of the graph.

Surprisingly, this combinatorial lemma (which, as we will see later, relies only on the $d$-dimensional $\Phi$-uniformity of $A$ and $B$ ) is quite difficult to prove. Although much of work needed for its proof was already done by Laczkovich [17], a rather long argument is still required to complete it, so we postpone all details to Section 4.3.

Given Lemma 4.2, another idea that went into the proof is the following. Given a partition of $\left(\mathbb{T}^{k}\right)_{\boldsymbol{u}} \cong \mathbb{Z}^{d}$ into a regular grid of $2^{j}$-cubes with a maximum matching inside each cube, group the cubes $2^{d}$ apiece so that the new groups form a $2^{j+1}$-regular grid. By Lemma 4.2 , the number of unmatched vertices inside each $2^{j}$-cube $Q$ is at most ||$A_{\boldsymbol{u}} \cap Q|-| B_{\boldsymbol{u}} \cap Q||$, which is at most $2 \Phi\left(2^{j}\right)$ by the assumptions of Theorem 2.2. In particular, the uniform density of unmatched points tends to 0 with $j \rightarrow \infty$, helping with (13). Inside each new $2^{j+1}$-cube $Q^{\prime}$, iteratively select and flip an augmenting path of length at most $2^{j+1}$ until none exists. By Lemma 4.2, we have a maximum matching inside $Q^{\prime}$ at the end. The total number of changed edges is at most $2^{j+1} \cdot 2^{d} \cdot 2 \Phi\left(2^{j}\right)$. If we iterate over all $j \in \mathbb{N}$ and sum the density of these changes, we get

$$
\sum_{j=0}^{\infty} \frac{2^{j+1} \cdot 2^{d} \cdot 2 \Phi\left(2^{j}\right)}{\left(2^{j+1}\right)^{d}}=4 \sum_{j=0}^{\infty} \frac{\Phi\left(2^{j}\right)}{2^{(d-1) j}} .
$$

The above sum converges by (5), giving a "coset analogue" of the desired requirement (14).

However, it is impossible to construct a perfect partition of each coset into cubes of the same side length $N \geqslant 2$ in an equivariant and measurable way (because the $\mathbb{Z}^{d}$-action on $\mathbb{T}^{k}$ given by the translations by $N \boldsymbol{x}_{1}, \ldots, N \boldsymbol{x}_{d}$ is ergodic for typical vectors $\boldsymbol{x}_{j}$ ). We overcome this issue by fixing, at each Iteration $i$, some set $S_{i} \subseteq \mathbb{T}^{k}$ such that the elements of $S_{i, \boldsymbol{u}} \subseteq \mathbb{Z}^{d}$ (called seeds) are far apart from each other. Informally speaking, we view each seed $s \in S_{i, \boldsymbol{u}}$ as a processor that "controls" its Voronoi cell; namely, $s$ draws the regular grid $\mathcal{Q}_{i}$ consisting of $N_{i}$-cubes inside its Voronoi cell, treating itself as the centre

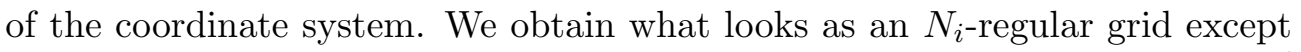
possible misalignments near cell boundaries. Also, assume that "most" of $\mathbb{Z}^{d}$ 
is already covered by grid-like areas of $N_{i-1}$-cubes with each cube containing a maximum matching that were constructed in the previous iteration step. Now, each $s \in S_{i, \boldsymbol{u}}$ aligns these as close as possible to its $N_{i}$-grid and then uses Lemma 4.2 to do incremental steps as in the previous paragraph, running them from $j=\log _{2} N_{i-1}$ to $\log _{2} N_{i}-1$ until every $N_{i}$-cube that is under control of $s$ induces a maximum matching. Of course, the possible misalignments of the grids and boundary issues require extra technical arguments. (This is the part where we need the $(d-1)$-dimensional $\Psi$-uniformity.)

We hope that the above discussion will be a good guide for understanding the proof of Part 1 of Theorem 2.2 which we present now.

4.2. Details of the proof. We will use the global constants that were defined at the beginning of Section 4.1.

4.2.1. Constructing the seed set $S_{i}$. Recall that a set $S \subseteq \mathbb{T}^{k}$ is $r$-sparse (given the free vectors $\boldsymbol{x}_{1}, \ldots, \boldsymbol{x}_{d}$ ) if for every $\boldsymbol{u} \in \mathbb{T}^{k}$ and every distinct $\boldsymbol{m}, \boldsymbol{n} \in$ $S_{\boldsymbol{u}}$ we have that $\|\boldsymbol{m}-\boldsymbol{n}\|_{\infty}>r$.

For each $i \in \mathbb{N}$, we construct a Borel set $S_{i} \subseteq \mathbb{T}^{k}$ which is maximal $N_{i+2}$-sparse, that is, $S_{i}$ is $N_{i+2}$-sparse but the addition of any new element of $\mathbb{T}^{k} \backslash S_{i}$ to it violates this property. (The maximality property will be useful in the proof of Lemma 4.3 as it will guarantee that the diameter of Voronoi cells of $S_{i, \boldsymbol{u}}$ is uniformly bounded.) Take the $N_{i+2}$-sparse map $\chi: \mathbb{T}^{k} \rightarrow[t]$ provided by Lemma 3.2. We construct $S_{i}$ by starting with the empty set and then, iteratively for $j \in[t]$, adding all those points of $\chi^{-1}(j)$ that do not violate the $N_{i+2}$-sparseness with an already existing element. Formally, we let $S_{i, 0}:=\emptyset$ and

$$
S_{i, j}:=S_{i, j-1} \cup\left(\chi^{-1}(j) \backslash \cup_{\boldsymbol{n} \in \operatorname{dist}_{\leqslant N}(\mathbf{2} 2}(\mathbf{0})\left(S_{i, j-1}+n_{1} \boldsymbol{x}_{1}+\ldots+n_{d} \boldsymbol{x}_{d}\right)\right),
$$

for $j=1, \ldots, t$. This formula shows, in particular, that the final set $S_{i}:=S_{i, t}$ is Borel. Also, $S_{i}$ is $N_{i+2}$-sparse (since each $\chi^{-1}(j)$ is) while the maximality of $S_{i}$ follows from the fact that each element $\boldsymbol{x} \in \mathbb{T}^{k}$ was considered for inclusion into the set $S_{i, \chi(\boldsymbol{x})} \subseteq S_{i}$.

4.2.2. Constructing grid domains around seeds. Here we construct an equivariant family $\left(\mathcal{Q}_{i, \boldsymbol{u}}\right)_{\boldsymbol{u} \in \mathbb{T}^{k}}$ consisting of disjoint $N_{i}$-cubes in $\mathbb{Z}^{d}$ that looks as the $N_{i}$-regular grid in a large neighbourhood of each point of $S_{i, u}$. This construction is similar to the one by Timár [36, 37], except he had to cover the whole space $\mathbb{Z}^{d}$ with parts that could somewhat deviate from being perfect cubes.

Let $\boldsymbol{u} \in \mathbb{T}^{k}$ and $\boldsymbol{s} \in S_{i, \boldsymbol{u}}$. Let the (integer) Voronoi cell of $\boldsymbol{s}$ be

$$
C_{i, \boldsymbol{s}, \boldsymbol{u}}:=\left\{\boldsymbol{n} \in \mathbb{Z}^{d}: \forall \boldsymbol{s}^{\prime} \in S_{i, \boldsymbol{u}} \backslash\{\boldsymbol{s}\} \quad\|\boldsymbol{n}-\boldsymbol{s}\|_{\infty}<\left\|\boldsymbol{n}-\boldsymbol{s}^{\prime}\right\|_{\infty}\right\},
$$

i.e. the set of points in $\mathbb{Z}^{d}$ strictly closer to $s$ than to any other element of $S_{i, \boldsymbol{u}}$. 
Since each element of $\mathbb{Z}^{d}$ is at distance at most $N_{i+2}$ from $S_{i, \boldsymbol{u}}$, we can "produce" Voronoi cells using some $N_{i+2}$-local rule $\mathcal{R}$. (Namely, we want $\mathcal{R}$ to transform the characteristic function of $S_{i}$ into the function whose value on every $\boldsymbol{u} \in \mathbb{T}^{k}$ encodes if there is $\boldsymbol{s} \in S_{i, \boldsymbol{u}}$ such that $C_{i, \boldsymbol{s}, \boldsymbol{u}}$ contains the origin and, if yes, stores such (unique) vector $s$.) In particular, the corresponding structure $C_{i}$ on $\mathbb{T}^{k}$ is measurable by Lemma 3.1 .

Let $\mathcal{Q}_{i, \boldsymbol{u}}$ consist of those $N_{i}$-cubes $Q=\prod_{j=1}^{d}\left[a_{j}, a_{j}+N_{i}-1\right]$ for which there is $\boldsymbol{s} \in S_{i, \boldsymbol{u}}$ such that $Q \subseteq C_{i, \boldsymbol{s}, \boldsymbol{u}}$ and all coordinates of the vector $\boldsymbol{a}-\boldsymbol{s}$ are divisible by $N_{i}$. Since integer Voronoi cells are disjoint, the constructed cubes are also disjoint. The following lemma states, in particular, that the set of vertices missed by these cubes is "small".

Lemma 4.3. Let $m \in \mathbb{N}$ be arbitrary and, for $\boldsymbol{u} \in \mathbb{T}^{k}$, let $X_{\boldsymbol{u}} \subseteq \mathbb{Z}^{d}$ be the set of points at $L^{\infty}$-distance at most $m$ from $\mathbb{Z}^{d} \backslash \cup \mathcal{Q}_{i, \boldsymbol{u}}$. Then the (equivariant) family $\left(X_{\boldsymbol{u}}\right)_{\boldsymbol{u} \in \mathbb{T}^{k}}$ has uniform density at most $O\left(\left(m+N_{i}\right) / N_{i+2}\right)$.

Proof. Fix $\boldsymbol{u} \in \mathbb{T}^{k}$. Let

$$
C_{i, \boldsymbol{s}, \boldsymbol{u}}^{\mathbb{R}}:=\left\{\boldsymbol{x} \in \mathbb{R}^{d}: \forall \boldsymbol{s}^{\prime} \in S_{i, \boldsymbol{u}}\|\boldsymbol{x}-\boldsymbol{s}\|_{\infty} \leqslant\left\|\boldsymbol{x}-\boldsymbol{s}^{\prime}\right\|_{\infty}\right\},
$$

be the real Voronoi cell of $s \in S_{i, \boldsymbol{u}}$. (The differences to the definition (16) are that now we consider any real vectors and we also include the boundary points.)

Take any $\boldsymbol{n} \in X_{\boldsymbol{u}}$. Let $\boldsymbol{s} \in S_{i, \boldsymbol{u}}$ be arbitrary such that the real cube $[0,1)^{d}+\boldsymbol{n}$ intersects $C_{i, \boldsymbol{s}, \boldsymbol{u}}^{\mathbb{R}}$. We know that $\boldsymbol{n}$ is at distance at most $m$ from

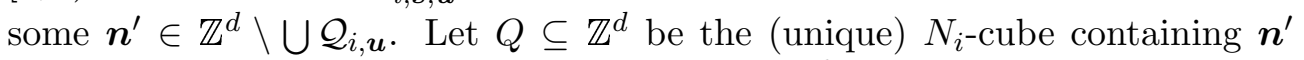
that $\boldsymbol{s}$ would have liked to claim (that is, $Q=\prod_{j=1}^{d}\left[a_{j}, a_{j}+N_{i}-1\right] \ni \boldsymbol{n}^{\prime}$ with each $a_{j}$ congruent to $s_{j}$ modulo $N_{i}$ ). Since $Q$ does not lie inside the integer Voronoi cell of $\boldsymbol{s}$, it has to contain a point $\boldsymbol{n}^{\prime \prime}$ which is not farther from some $\boldsymbol{s}^{\prime} \in S_{i, \boldsymbol{u}} \backslash\{\boldsymbol{s}\}$ than from $\boldsymbol{s}$. Thus $\operatorname{dist}(\boldsymbol{n}, Y) \leqslant\left\|\boldsymbol{n}-\boldsymbol{n}^{\prime \prime}\right\|_{\infty} \leqslant m+N_{i}-1$, where $Y$ is the closure of $\mathbb{R}^{d} \backslash C_{i, \boldsymbol{s}, \boldsymbol{u}}^{\mathbb{R}}$. It follows that every element of $[0,1)^{d}+\boldsymbol{n}$ is at distance at most $m+N_{i}$ from $Y$.

On the other hand, by the $N_{i+2}$-spareness of $S_{i, \boldsymbol{u}}$ the distance between $\boldsymbol{s}$ and $Y$ is larger than $N_{i+2} / 2$. Thus, if we shrink $C_{i, \boldsymbol{s}, \boldsymbol{u}}^{\mathbb{R}}$ by factor $\gamma:=$ $\left(N_{i+2}-2 m-2 N_{i}\right) / N_{i+2}$ from $\boldsymbol{s}$, i.e. we take the set $\gamma C_{i, \boldsymbol{s}, \boldsymbol{u}}^{\mathbb{R}}+(1-\gamma) \boldsymbol{s} \subseteq C_{i, \boldsymbol{s}, \boldsymbol{u}}^{\mathbb{R}}$, then it will be disjoint from $[0,1)^{d}+\boldsymbol{n}$. It follows that the set $[0,1)^{d}+X_{\boldsymbol{u}}:=$ $\cup_{\boldsymbol{n} \in X_{\boldsymbol{u}}}\left([0,1)^{d}+\boldsymbol{n}\right)$ can cover at most $1-\gamma^{d}$ fraction of the volume of any Voronoi cell $C_{i, \boldsymbol{s}, \boldsymbol{u}}^{\mathbb{R}}$.

By the maximality of $S_{i, \boldsymbol{u}} \subseteq \mathbb{Z}^{d}$ (and since any point of $\mathbb{R}^{d}$ is at distance at most $1 / 2$ from $\mathbb{Z}^{d}$ ), the distance between $s \in S_{i, \boldsymbol{u}}$ and any point on the boundary of $C_{i, \boldsymbol{s}, \boldsymbol{u}}^{\mathbb{R}}$ is at most $N_{i+2}+1 / 2$. Thus the real Voronoi cells, that cover the whole space, have a uniformly bounded diameter. It follows that 
there is a constant $N$ (independent of $\boldsymbol{u}$ ) such that the density of $X_{\boldsymbol{u}}$ inside any $N$-cube is at most, say, $2\left(1-\gamma^{d}\right)=O\left(\left(m+N_{i}\right) / N_{i+2}\right)$, as required.

4.2.3. Constructing the matchings $\mathcal{M}_{i}$. Iteratively for $i=0,1, \ldots$, we will construct a measurable matching $\mathcal{M}_{i}$ in $\mathcal{G}$ (or, equivalently, an equivariant and measurable family $\left(\mathcal{M}_{i, \boldsymbol{u}}\right)_{\boldsymbol{u} \in \mathbb{T}^{k}}$ where $\mathcal{M}_{i, \boldsymbol{u}}$ is a matching in $\left.\mathcal{G}_{\boldsymbol{u}}\right)$ such that the following two properties hold for each $\boldsymbol{u} \in \mathbb{T}^{k}$ :

(1) every edge of $\mathcal{M}_{i, \boldsymbol{u}}$ lies inside some cube $Q \in \mathcal{Q}_{i, \boldsymbol{u}}$ (that is, $\mathcal{M}_{i, \boldsymbol{u}} \subseteq$ $\left.\cup_{Q \in \mathcal{Q}_{i, u}} Q^{2}\right)$

(2) for every cube $Q \in \mathcal{Q}_{i, \boldsymbol{u}}$ the restriction of $\mathcal{M}_{i, \boldsymbol{u}}$ to $Q$ (more precisely, to the induced bipartite subgraph $\mathcal{G}_{\boldsymbol{u}}[Q, Q]$ ) is a maximum matching.

For $i=0$, we construct $\mathcal{M}_{0, \boldsymbol{u}}$ by taking a maximum matching inside each cube $Q \in \mathcal{Q}_{0, \boldsymbol{u}}$. In order to make it equivariant and measurable we consistently use some local rule: for example, take the lexicographically smallest maximum matching in $Q$, with respect to the natural labelling of the $N_{0}$-cube $Q$ by $\left[N_{0}\right]^{d}$.

Let us explain how this can be realised by a local rule $\mathcal{R}$ of radius $2 N_{0}+N_{2}$. The rule transforms the function $g: \mathbb{T}^{k} \rightarrow \mathbb{N}$ that encodes the triple of sets $\left(A, B, S_{0}\right)$ into one that encodes $\mathcal{M}_{0}$. Take any $\boldsymbol{a} \in A$. Note that the restriction of $g_{\boldsymbol{a}}$ to $Q^{\prime}:=\left[-2 N_{0}-N_{2}, 2 N_{0}+N_{2}\right]^{d}$ determines $A_{\boldsymbol{a}} \cap Q^{\prime}, B_{\boldsymbol{a}} \cap Q^{\prime}$ and $S_{0, \boldsymbol{a}} \cap Q^{\prime}$. Since $S_{0, \boldsymbol{a}}$ is maximal $N_{2}$-sparse, we have that $S_{0, \boldsymbol{a}} \cap\left[-N_{2}, N_{2}\right]^{d} \neq \emptyset$. From the latter set, take a point $s$ which is closest to the origin $\mathbf{0}$. Let $Q \subseteq \mathbb{Z}^{d}$ be the $N_{0}$-cube that contains $\mathbf{0}$ and belongs to the $N_{0}$-grid centred at $\boldsymbol{s}$. We have that $Q \in \mathcal{Q}_{0, \boldsymbol{a}}$ if and only if every element of $Q$ is closer to $s$ than to $S_{0, \boldsymbol{a}} \backslash\{\boldsymbol{s}\}$. We see that all elements of $S_{0, \boldsymbol{a}}$ that can "interfere" with $Q$ are at distance at most $N_{0}+N_{2}$ from $Q$ and thus are confined to $Q^{\prime}$. Therefore, the set $S_{0, \boldsymbol{a}} \cap Q^{\prime}$ determines the $N_{0}$-cube of $\mathcal{Q}_{0, \boldsymbol{a}}$ containing $\mathbf{0}$, if it exists (which has to be $Q$ then). Suppose that $Q \in \mathcal{Q}_{0, a}$. Since $Q$ is a subset of $Q^{\prime}$, we know the intersections of $A_{\boldsymbol{a}}$ and $B_{\boldsymbol{a}}$ with $Q$. Now, among all (finitely many) maximum matchings in $\mathcal{G}_{\boldsymbol{a}}\left[A_{\boldsymbol{a}} \cap Q, B_{\boldsymbol{a}} \cap Q\right]$, take the lexicographically smallest matching $\mathcal{M} \subseteq Q^{2}$. If $\mathcal{M}$ matches $\mathbf{0} \in A_{\boldsymbol{a}}$, then the local rule outputs $\boldsymbol{n}:=\mathcal{M}(\mathbf{0})$, which tells us that the $\mathcal{M}_{0}$-match of $\boldsymbol{a} \in A$ is $\boldsymbol{a}+\sum_{i=1}^{d} n_{i} \boldsymbol{x}_{i}$. (This is an element of $B$ since $\boldsymbol{n} \in B_{\boldsymbol{a}}$.) Otherwise (namely, if $\boldsymbol{a} \notin A$, or $Q \notin \mathcal{Q}_{0, \boldsymbol{a}}$, or $\mathcal{M}(\mathbf{0})$ is undefined) the local rule outputs that $\boldsymbol{a}$ is not matched. The obtained matching $\mathcal{M}_{0}$ is measurable by Lemma 3.1 , since each of the sets $A, B, S_{0} \subseteq \mathbb{T}^{k}$ is measurable. From now on, we may omit details like this.

Let $i \geqslant 1$ and suppose that we have $\mathcal{M}_{i-1}$ satisfying all above properties. Let us describe how to construct $\mathcal{M}_{i}$. We will do this in three steps, producing intermediate matchings $\mathcal{M}_{i}^{\prime}$ and $\mathcal{M}_{i}^{\prime \prime}$. For each step, we also provide an upper bound on the measure of vertices in $A$ that undergo some change; these estimates will be later used to argue that (14) holds. So, take any $\boldsymbol{u} \in \mathbb{T}^{k}$. 
Let $\mathcal{M}_{i, \boldsymbol{u}}^{\prime}$ consist of those edges $(\boldsymbol{m}, \boldsymbol{n}) \in \mathcal{M}_{i-1, \boldsymbol{u}}$ for which there is a cube $Q \in \mathcal{Q}_{i, \boldsymbol{u}}$ with $\boldsymbol{m}, \boldsymbol{n} \in Q$. In other words, when we pass from $\mathcal{M}_{i-1}$ to $\mathcal{M}_{i}^{\prime}$, we discard all previous edges that have at least one vertex in $X:=\mathbb{T}^{k} \backslash \cup \mathcal{Q}_{i}$ or connect two different cubes of $\mathcal{Q}_{i}$. By Lemma 4.3, the set $X$ has uniform density at most $O\left(N_{i} / N_{i+2}\right)$ while, trivially, points within distance $M$ from the boundary of some $\mathcal{Q}_{i, \boldsymbol{u}}$-cube have uniform density $O\left(1 / N_{i}\right)$. Thus, by Lemma 4.1 , when we pass from $\mathcal{M}_{i-1}$ to $\mathcal{M}_{i}^{\prime}$, we change the current matching on a set of measure

$$
\lambda\left(\left(\mathcal{M}_{i-1} \triangle \mathcal{M}_{i}^{\prime}\right)^{-1}(B)\right)=O\left(N_{i} / N_{i+2}+1 / N_{i}\right) .
$$

Next, let $\mathcal{M}_{i, \boldsymbol{u}}^{\prime \prime}$ be obtained by modifying $\mathcal{M}_{i, \boldsymbol{u}}^{\prime}$ as follows. For every cube $Q \in \mathcal{Q}_{i, \boldsymbol{u}}$ that has at least one vertex that lies outside of $\bigcup \mathcal{Q}_{i-1, \boldsymbol{u}}$ or has vertices that come from different integer Voronoi cells of $S_{i-1, \boldsymbol{u}}$, let the restriction of $\mathcal{M}_{i, \boldsymbol{u}}^{\prime \prime}$ to $Q$ be any maximum matching. (Thus we completely ignore $\mathcal{M}_{i, \boldsymbol{u}}^{\prime}$ inside such cubes $Q$.) Lemma 4.3 (applied to $i-1$ and $m=N_{i}$ ) shows that the union of such cubes $Q$ has uniform density $O\left(N_{i} / N_{i+1}\right)$. Thus by Lemma 4.1, we have that

$$
\lambda\left(\left(\mathcal{M}_{i}^{\prime} \triangle \mathcal{M}_{i}^{\prime \prime}\right)^{-1}(B)\right)=O\left(N_{i} / N_{i+1}\right) .
$$

Finally, we have to show how to obtain the desired matching $\mathcal{M}_{i}$ by modifying $\mathcal{M}_{i}^{\prime \prime}$ on the remaining cubes, so that the new matching is maximum inside each $\mathcal{Q}_{i, \boldsymbol{u}}$-cube. Let $Q \in \mathcal{Q}_{i, \boldsymbol{u}}$ be one of the remaining cubes. This means that $Q$ lies entirely inside the Voronoi cell $C_{i, \boldsymbol{s}, \boldsymbol{u}}$ of some $s \in S_{i-1, \boldsymbol{u}}$ and is completely covered by $N_{i-1}$-cubes from $\mathcal{Q}_{i-1, \boldsymbol{u}}$. These cubes when restricted to $Q$ make a regular grid that, however, need not be properly aligned with the sides of $Q$. Take $j \in[d]$. Let $I_{j} \subseteq \mathbb{Z}$ be the projection of $Q$ onto the $j$-th axis and let $I_{j, 0} \cup \ldots \cup I_{j, t_{j}}$ be the partition of $I_{j}$ into consecutive intervals given by the grid. Each $t_{j}$ is $2^{h}-1$ or $2^{h}$, where

$$
h:=\log _{2}\left(N_{i} / N_{i-1}\right) .
$$

(Since $N_{i-1}<N_{i}$ are powers of $2, h$ is a positive integer.) If $t_{j}=2^{h}$, then we change the partition of $I_{j}$ as follows. By reversing the order of the second index if necessary, assume that $\left|I_{j, t_{j}}\right| \leqslant N_{i-1} / 2$ (note that $\left|I_{j, 0}\right|+\left|I_{j, t_{j}}\right|=$ $N_{i-1}$ ), merge $I_{j, t_{j}}$ into $I_{j, t_{j}-1}$ (with the new interval still denoted as $I_{j, t_{j}-1}$ ), and decrease $t_{j}$ by 1 . After we perform this merging for all $j$ with $t_{j}=2^{h}$, the obtained interval partitions induce a new grid on $Q$ that splits it into $2^{h d}$ rectangles. We call these rectangles basic and naturally index them by $\left(\{0,1\}^{d}\right)^{h}$. Namely, for $\boldsymbol{B}=\left(\boldsymbol{b}_{1}, \ldots, \boldsymbol{b}_{h}\right)$ with each $\boldsymbol{b}_{t}$ being a binary sequence of length $d$, let $R^{B}:=\prod_{j=1}^{d} I_{j, s_{j}}$, where $s_{j} \in\left[0,2^{h}-1\right]$ is the integer whose base-2 representation has digits $\left(b_{1, j}, \ldots, b_{h, j}\right)$. Furthermore, if $\left(\boldsymbol{b}_{1}, \ldots, \boldsymbol{b}_{t}\right) \in\left(\{0,1\}^{d}\right)^{t}$ 

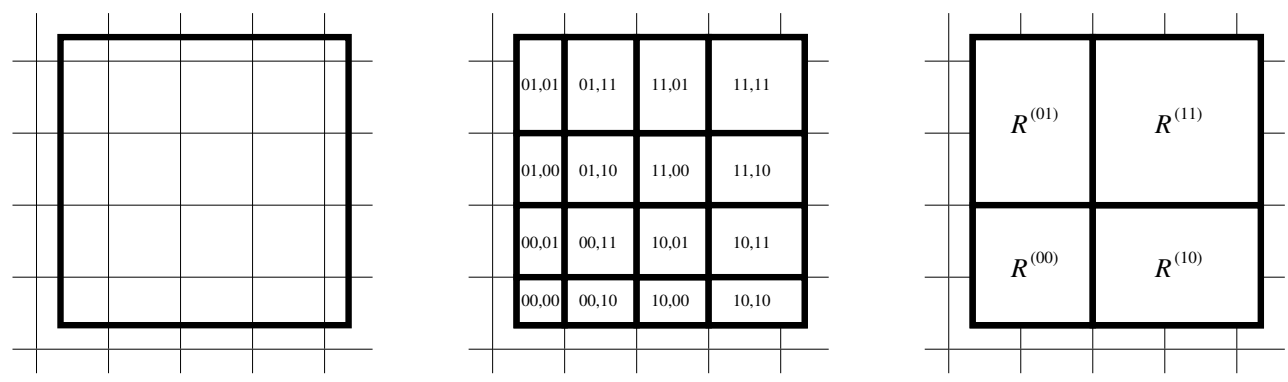

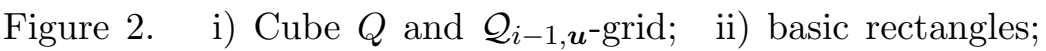

iii) level-1 rectangles

with $t<h$, then we define

$$
R^{\left(\boldsymbol{b}_{1}, \ldots, \boldsymbol{b}_{t}\right)}:=\cup_{\left(\boldsymbol{b}_{t+1}, \ldots, \boldsymbol{b}_{h}\right) \in\left(\{0,1\}^{d}\right)^{h-t}} R^{\left(\boldsymbol{b}_{1}, \ldots, \boldsymbol{b}_{h}\right)}
$$

to be the union of those basic rectangles whose index sequence has $\left(\boldsymbol{b}_{1}, \ldots, \boldsymbol{b}_{t}\right)$ as a prefix. Thus, the largest (or level-0) rectangle is $R^{()}=Q$, which splits into $2^{d}$ level-1 rectangles as $R^{()}=\cup_{\boldsymbol{b} \in\{0,1\}^{d}} R^{(\boldsymbol{b})}$, each of which splits further as $R^{(\boldsymbol{b})}=\cup_{\boldsymbol{a} \in\{0,1\}^{d}} R^{(\boldsymbol{b}, \boldsymbol{a})}$, and so on until we get the basic rectangles at level $h$. See Figure 2 for an illustration with $d=h=2$.

Note that, for each $j \in[0, h]$, a level- $j$ rectangle has side lengths between $2^{h-j} N_{i-1} \pm N_{i-1} / 2$; in particular it is 3-balanced. Also, $\mathcal{M}_{i, \boldsymbol{u}}^{\prime \prime} \subseteq \mathcal{M}_{i-1, \boldsymbol{u}}$ cannot connect two different basic rectangles. Since $\mathcal{M}_{i, \boldsymbol{u}}^{\prime \prime} \cap Q^{2}=\mathcal{M}_{i-1, \boldsymbol{u}} \cap Q^{2}$, it is a maximum matching on every basic rectangle which is an element of $\mathcal{Q}_{i-1, \boldsymbol{u}}$.

We are now ready to describe how we modify $\mathcal{M}_{i, \boldsymbol{u}}^{\prime \prime}$ on $Q$. First, put an arbitrary maximum matching on every basic rectangle not in $\mathcal{Q}_{i-1, \boldsymbol{u}}$. (For example, in Figure 2.ii these happen to be all 12 rectangles at the boundary of $Q$.) This involves at most $2 N_{i-1} \cdot d N_{i}^{d-1}$ elements of $Q$ and thus has uniform density at most $O\left(N_{i-1} / N_{i}\right)$. Next, we iteratively repeat the following for $\ell=h, \ldots, 1$. Suppose that the current matching is maximum when restricted to each level- $\ell$ rectangle (i.e. to each $R^{\left(\boldsymbol{b}_{1}, \ldots, \boldsymbol{b}_{\ell}\right)}$ ) and does not connect two such rectangles. (Note that this is the case at the initial step $\ell=h$.) Inside each level- $(\ell-1)$ rectangle $R$, iteratively augment the current matching using paths of length at most $\left(2^{h-\ell+1}+1 / 2\right) N_{i-1}$ until none remains. Clearly, each augmentation increases the size of the matching inside the finite set $R$, so we run out of augmenting paths after finitely many flips. By Lemma 4.2, the final matching in $\mathcal{G}_{\boldsymbol{u}}[R, R]$ covers all vertices in one part. In particular, it is maximum (and we can proceed with the next value of $\ell$ ).

Note that the number of unmatched vertices in any level- $(\ell-1)$ rectangle $R^{\boldsymbol{B}}, \boldsymbol{B} \in\left(\{0,1\}^{d}\right)^{\ell-1}$, before Iteration $\ell$ was at most $\sum_{\boldsymbol{b} \in\{0,1\}^{d}} D\left(R^{(\boldsymbol{B}, \boldsymbol{b})}\right)$, 
where

$$
D(Y):=|| A_{\boldsymbol{u}} \cap Y|-| B_{\boldsymbol{u}} \cap Y||
$$

denotes the $\left(A_{\boldsymbol{u}}, B_{\boldsymbol{u}}\right)$-discrepancy of a finite set $Y \subseteq \mathbb{Z}^{d}$. (This holds because, again by Lemma 4.2 , a maximum matching inside $R^{(\boldsymbol{B}, \boldsymbol{b})}$ for each $\boldsymbol{b} \in\{0,1\}^{d}$ has to match one part completely.) Thus, when we pass from $\mathcal{M}_{i}^{\prime \prime}$ to $\mathcal{M}_{i}$, the density of changes inside $Q$ caused by augmenting paths is at most

$$
\frac{1}{|Q|} \sum_{\ell=1}^{h} O\left(N_{i} / 2^{\ell}\right) \sum_{\boldsymbol{B} \in\left(\{0,1\}^{d}\right)^{\ell}} D\left(R^{\boldsymbol{B}}\right) .
$$

Call a level- $\ell$ rectangle $R$ special if at least two of its side lengths are different from $N_{i} / 2^{\ell}$. (For example, in Figure 2.ii the special rectangles happen to be all four corner rectangles.)

The $\left(A_{\boldsymbol{u}}, B_{\boldsymbol{u}}\right)$-discrepancy of a non-special level- $\ell$ rectangle $R$ can be bounded by decomposing it into $(d-1)$-dimensional $N_{i} / 2^{\ell}$-cubes and using the $\Psi$-uniformity of both $A_{\boldsymbol{u}}$ and $B_{\boldsymbol{u}}$ :

$$
D(R) \leqslant 2 \Psi\left(N_{i} / 2^{\ell}\right) \frac{|R|}{\left(N_{i} / 2^{\ell}\right)^{d-1}} .
$$

Thus such rectangles contribute $O\left(\Psi\left(N_{i} / 2^{\ell}\right) /\left(N_{i} / 2^{\ell}\right)^{d-2}\right)$ to (20).

We bound the $\left(A_{\boldsymbol{u}}, B_{\boldsymbol{u}}\right)$-discrepancy of a special level- $\ell$ rectangle $R$ using the following argument with $N:=N_{i} / 2^{\ell}$ and $n:=N_{i-1} / 2$. For notational convenience, assume that $R=\prod_{j=1}^{d}\left[r_{j}\right]$ (thus $\left|r_{j}-N\right| \leqslant n$ for each $j \in[d]$ ) and that $r_{j} \geqslant N$ exactly for $j \in[t]$. For $j \in[t]$ (resp. $j \in[t+1, d]$ ) let $R_{j}$ be the rectangle which is the product of $d$ copies of $[N]$ except the $j$-th factor is $\left[N+1, r_{j}\right]$ (resp. $\left[r_{j}+1, N\right]$ ). We can transform $[N]^{d}$ into $R$ by adding the rectangles $R_{1}, \ldots, R_{t}$, then subtracting $R_{t+1}, \ldots, R_{d}$, and finally adjusting those vertex multiplicities that are still wrong. The last step involves at most $\left(\begin{array}{l}d \\ 2\end{array}\right) n^{2} N^{d-2}$ vertices of $R$ and each multiplicity has to be adjusted by at most $d$. Thus we have the following Bonferroni-type inequality:

$$
D(R) \leqslant D\left([N]^{d}\right)+\sum_{j=1}^{d} D\left(R_{j}\right)+d\left(\begin{array}{l}
d \\
2
\end{array}\right) n^{2} N^{d-2}=O\left(N \Psi(N)+n^{2} N^{d-2}\right) .
$$

Also, the total number of special rectangles at level $\ell$ is at most $O\left(2^{\ell(d-2)}\right)$. Indeed, we have at most $4\left(\begin{array}{l}d \\ 2\end{array}\right)$ ways to choose a " $(d-2)$-dimensional face" $F$ of $Q$, and then observe that $F$ intersects at most $2^{\ell(d-2)}$ level- $\ell$ rectangles (while each special rectangle must have a non-empty footprint in at least one $(d-2)$-dimensional face of $Q)$.

Thus, special level- $\ell$ rectangles contribute $O\left(4^{-\ell} \Psi\left(N_{i} / 2^{\ell}\right) /\left(N_{i} / 2^{\ell}\right)^{d-2}+\right.$ $\left.2^{-\ell} N_{i-1}^{2} / N_{i}\right)$ to $(20)$. 
Putting all together and using Lemma 4.1, we get the following upper bound on the measure of points where $\mathcal{M}_{i}^{\prime \prime}$ and $\mathcal{M}_{i}$ differ:

$$
\lambda\left(\left(\mathcal{M}_{i}^{\prime \prime} \triangle \mathcal{M}_{i}\right)^{-1}(B)\right)=O\left(N_{i-1}^{2} / N_{i}\right)+O(1) \sum_{\ell=1}^{h} \frac{\Psi\left(N_{i} / 2^{\ell}\right)}{\left(N_{i} / 2^{\ell}\right)^{d-2}} .
$$

Having constructed $\mathcal{M}_{i}$, we increase $i$ by one and repeat the above procedure. It remains to show that the constructed sequence of measurable matchings $\left(\mathcal{M}_{i}\right)_{i \in \mathbb{N}}$ has the required properties.

Observe that each upper bound in (17), (18), and (21) is a summable function of $i \in \mathbb{N}$. This directly follows from our choice of the sequence $\left(N_{i}\right)_{i \in \mathbb{N}}$, with the exception of the second term in the right-hand side of (21). Here, if we sum these terms over $i \in \mathbb{N}$, then each integer power $2^{j}$ can appear as $N_{i} / 2^{\ell}$ at most once (indeed, $i \in \mathbb{N}$ has to be the unique index such that $N_{i-1} \leqslant 2^{j}<N_{i}$ ); thus the resulting sum converges by (5). Now (14) follows, since

$$
\mathcal{M}_{i-1} \triangle \mathcal{M}_{i} \subseteq\left(\mathcal{M}_{i-1} \triangle \mathcal{M}_{i}^{\prime}\right) \cup\left(\mathcal{M}_{i}^{\prime} \triangle \mathcal{M}_{i}^{\prime \prime}\right) \cup\left(\mathcal{M}_{i}^{\prime \prime} \triangle \mathcal{M}_{i}\right) .
$$

Since $\mathcal{M}_{i, \boldsymbol{u}}$ covers all but at most $D(Q) \leqslant 2 \Phi\left(N_{i}\right)$ vertices inside each cube in $\mathcal{Q}_{i, \boldsymbol{u}}$ while the set of vertices not covered by $\mathcal{Q}_{i}$ has uniform density $O\left(N_{i} / N_{i+2}\right)$ by Lemma 4.3 , the set $A \backslash \mathcal{M}_{i}^{-1}(B)$ has uniform density at most $O\left(\Phi\left(N_{i}\right) / N_{i}^{d}+N_{i} / N_{i+2}\right)$. Clearly, this tends to zero as $i \rightarrow \infty$. Thus, by Lemma 4.1, the other desired estimate (13) also holds.

The proof of Part 1 of Theorem 2.2 can now be completed, as it was described after (14).

4.3. Proof of Lemma 4.2. This section is dedicated to proving Lemma 4.2 that was needed in the proof of Part 1 of Theorem 2.2. Note that Lemma 4.2 is a combinatorial statement that does not involve any notion of equivariance or measurability.

For $X, R \subseteq \mathbb{Z}^{d}$, define the internal boundary of $X$ relative to $R$ to be

$$
\partial^{R} X:=(\partial X) \cap R^{2}=\left\{(\boldsymbol{m}, \boldsymbol{n}) \in R^{2}: \boldsymbol{m} \in X, \boldsymbol{n} \notin X\right\}
$$

and the internal perimeter of $X$ relative to $R$ to be $p^{R}(X):=\left|\partial^{R} X\right|$. Recall that a rectangle $R \subseteq \mathbb{Z}^{d}$ is called $\rho$-balanced if the ratio of any its two side lengths is at most $\rho$.

Our next lemma states that a positive fraction of the boundary of a set $X$ lying inside a $\rho$-balanced rectangle $R$ is internal, unless $X$ occupies most of $R$.

Lemma 4.4. For every $R, X \subseteq \mathbb{Z}^{d}$, where $X \subseteq R$ and $R$ is a $\rho$-balanced rectangle, we have that

$$
p^{R}(X) \geqslant \frac{p(X)}{3 d \rho} \cdot \frac{|R \backslash X|}{|R|} .
$$


Proof. Let $\gamma:=d \rho / 2+2 d+1$, which is at most $3 d \rho$ since $d \geqslant 2$ and $\rho \geqslant 1$. We can assume that $p^{R}(X)<p(X) / \gamma$ for otherwise we are trivially done. Let $j \in[d]$ be such that at least $1 / d$-fraction of the common boundary $\partial X \cap \partial R$ goes in the $j$-th coordinate direction. Thus the number of lines parallel to the $j$-th coordinate axis that intersect $X$ is at least half of this quantity. Trivially, at most $p^{R}(X)$ of these lines can intersect $R \backslash X$ while every other line contains $r_{j}$ points from $X$, where $r_{1}, \ldots, r_{d}$ are the side lengths of $R$. Thus

$$
|X| \geqslant r_{j}\left(\frac{p(X)-p^{R}(X)}{2 d}-p^{R}(X)\right) \geqslant \frac{r_{j}(\gamma-2 d-1)}{2 d \gamma} p(X) .
$$

For every pair $(\boldsymbol{a}, \boldsymbol{b}) \in X \times(R \backslash X)$ consider the path inside $R$ which is the union of the $d$ "straight-line" paths that connect the following $d+1$ points in the stated order:

$$
\boldsymbol{a}=\left(a_{1}, \ldots, a_{d}\right), \quad\left(b_{1}, a_{2}, \ldots, a_{d}\right), \quad\left(b_{1}, b_{2}, a_{3}, \ldots, a_{d}\right), \quad \ldots, \quad \boldsymbol{b}=\left(b_{1}, \ldots, b_{d}\right) .
$$

Each such path contains at least one pair from $\partial^{R} X$. Conversely, every (ordered) pair in the internal boundary of $X$ going, for example, in the $i$-th direction is in at most $\left(r_{i}^{2} / 4\right) \prod_{h \in[d] \backslash\{i\}} r_{h}=\left(r_{i} / 4\right)|R|$ such paths. Denoting $r:=\max \left\{r_{i}: i \in[d]\right\}$ and using $(22)$, we conclude that

$$
p^{R}(X) \geqslant \frac{|X| \cdot|R \backslash X|}{(r / 4)|R|} \geqslant \frac{2(\gamma-2 d-1) p(X)}{d \rho \gamma} \cdot \frac{|R \backslash X|}{|R|} .
$$

Using that $\gamma \leqslant 3 d \rho$ satisfies $2(\gamma-2 d-1)=d \rho$, we obtain the required bound.

For a real $\delta \geqslant 0$ and sets $X, R \subseteq \mathbb{Z}^{d}$ such that $R$ is finite, let the discrepancy of $X$ relative to $R$ of density $\delta$ be

$$
D_{\delta}(X ; R):=|| X \cap R|-\delta| R|| .
$$

Thus, a set $X \subseteq \mathbb{Z}^{d}$ is $\Phi$-uniform of density $\delta$ if and only if $D_{\delta}(X ; Q) \leqslant \Phi\left(2^{i}\right)$ for every $2^{i}$-cube $Q \subseteq \mathbb{Z}^{d}$.

Clearly, Lemma 4.2 follows from Part 2 of the following result when applied to $\mathrm{A}:=A_{\boldsymbol{u}}$ and $\mathrm{B}:=B_{\boldsymbol{u}}$, assuming that $M$ satisfies Lemma 4.5 for $\rho:=3$ (with $d \in \mathbb{N}, \delta>0$, and $\Phi\left(2^{i}\right)=2^{i} \Psi\left(2^{i}\right)$ being as in Theorem 2.2).

Lemma 4.5. For every integer $d \geqslant 1$, reals $\delta>0$ and $\rho \geqslant 1$, and a function $\Phi:\left\{2^{i}: i \in \mathbb{N}\right\} \rightarrow \mathbb{R}$ satisfying $\sum_{i=0}^{\infty} \Phi\left(2^{i}\right) / 2^{(d-1) i}<\infty$, there is a constant $M=M(d, \delta, \rho, \Phi)$ such that the following holds for every $\rho$-balanced rectangle $R:=\prod_{j=1}^{d}\left[a_{j}, a_{j}+r_{j}-1\right] \subseteq \mathbb{Z}^{d}$ and sets $\mathrm{A}, \mathrm{B} \subseteq \mathbb{Z}^{d}$ that are $\Phi$-uniform of density $\delta>0$.

If $N:=\max \left(r_{1}, \ldots, r_{d}\right)$ is the maximum side length of $R$ and

$$
\mathcal{F}:=(\mathrm{A} \cap R, \mathrm{~B} \cap R, E)
$$


is the bipartite graph with edge set $E:=\{(\boldsymbol{m}, \boldsymbol{n}) \in(\mathrm{A} \cap R) \times(\mathrm{B} \cap R)$ : $\left.\|\boldsymbol{m}-\boldsymbol{n}\|_{\infty} \leqslant M\right\}$, then

(1) for every $X \subseteq \mathrm{A} \cap R$, we have

$$
|E(X)| \geqslant \min \left(|X|+10 d \cdot|X|^{(d-1) / d}, \frac{|\mathrm{B} \cap R|}{2}\right),
$$

where $E(X)=\{y: \exists x \in X(x, y) \in E\}$ is the neighbourhood of $X$ in $\mathcal{F}$;

(2) for every matching $\mathcal{M}$ in $\mathcal{F}$ that leaves unmatched elements in both parts, there is an augmenting path of length at most $N$.

Proof. Given $d, \delta, \rho$, and $\Phi$, choose sufficiently large integers in the order $M_{0} \ll M_{1} \ll M$.

Since $\sum_{i=0}^{\infty} \Phi\left(2^{i}\right) / 2^{(d-1) i}<\infty$, Theorem 1.2 in Laczkovich [17] shows that, for every $X \subseteq \mathbb{Z}^{d}$ which is $\Phi$-uniform of density $\delta$, we have

$$
D_{\delta}(X ; Y) \leqslant M_{0} p(Y), \quad \text { for all finite } Y \subseteq \mathbb{Z}^{d} .
$$

Note that the coefficient $M_{0}=M_{0}(d, \delta, \rho, \Phi)$ in (23) does not depend on the choice of $X$ and $Y$. (The proof of (23) in [17] proceeds by representing each $Y$ as a certain combination of binary cubes having the appropriately defined "complexity" at most $M_{0} p(Y)$.)

Next, take arbitrary A, B, $R \subseteq \mathbb{Z}^{d}$ as in the statement of the lemma. Assume that $N>M$ for otherwise $\mathcal{F}$ is a complete bipartite graph and the lemma trivially holds.

We prove Part 1 of the lemma first. Fix an arbitrary set $X \subseteq \mathrm{A} \cap R$ with $2|E(X)|<|\mathrm{B} \cap R|$.

Very roughly, the proof proceeds as follows. We define some "smoothed" versions $X_{1}$ and $X_{2}$ of $X$, where for illustration purposes one can imagine $X_{i}$ as $\operatorname{dist}_{\leqslant i M_{1}}(X) \subseteq \mathbb{Z}^{d}$, the $\left(i M_{1}\right)$-ball around $X$. Then every point of $\mathrm{B} \cap X_{2}$ is a neighbour of $X$. Also, if the boundaries of $X_{1}$ and $X_{2}$ are "smooth on the scale of $M_{1}$ ", then we expect that $\left|X_{2} \backslash X_{1}\right| \geqslant \Omega\left(M_{1}\left(p\left(X_{1}\right)+p\left(X_{2}\right)\right)\right)$. On the other hand, by (23) the number of points from A and B inside $X_{i}$ deviates from the expected value $\delta\left|X_{i}\right|$ by at most $M_{0} p\left(X_{i}\right)$ which is much smaller than $M_{1} p\left(X_{i}\right)$. This suffices to get the desired gap between $|E(X)| \geqslant\left|\mathrm{B} \cap X_{2}\right|$ and $|X| \leqslant\left|\mathrm{A} \cap X_{1}\right|$. The above argument is from Laczkovich [17]. However, here we have to confine all sets to $R$ (even if $X$ comes very close to the boundary of $R$ ). With an appropriate definition of $X_{i}$, the above argument can be applied if $\left|X_{i}\right|<\frac{2}{3}|R|$ as then $X_{i}$ has a positive fraction of its boundary in the interior of $R$ by Lemma 4.4. Otherwise there is a large discord between $\left|X_{i}\right| \geqslant \frac{2}{3}|R|$ and $\left|\mathrm{B} \cap X_{i}\right| \leqslant|E(X)|<|\mathrm{B} \cap R| / 2$, implying that $p\left(X_{i}\right)=\Omega\left(N^{d}\right)$; thus $p^{R}\left(X_{i}\right) \geqslant p\left(X_{i}\right)-p(R)=p\left(X_{i}\right)-O\left(N^{d-1}\right)$ is essentially the same as $p\left(X_{i}\right)$ and the above argument still applies. Let us give all details now. 
For each $j \in[d]$, fix a partition $\left[a_{j}, a_{j}+r_{j}-1\right]=\cup_{i=1}^{t_{j}} I_{j, i}$ of the $j$-th side of $R$ into intervals of length $M_{1}$ and $M_{1}+1$. (For example, take $r_{j}\left(\bmod M_{1}\right)$ intervals of length $M_{1}+1$ that occupy at most $\left(M_{1}-1\right)\left(M_{1}+1\right) \leqslant M / \rho \leqslant r_{j}$ initial elements and split the rest into intervals of length $M_{1}$.) Call each $d$ dimensional product $\prod_{j=1}^{d} I_{j, s_{j}}$ with $s \in \prod_{j=1}^{d}\left[t_{j}\right]$ a sub-rectangle. Thus we have partitioned $R$ into an (almost regular) grid made of sub-rectangles. We say that two sets $Y, Z \subseteq \mathbb{Z}^{d}$ share boundary if there is $(\boldsymbol{m}, \boldsymbol{n}) \in \partial Y$ such that $(\boldsymbol{n}, \boldsymbol{m}) \in \partial Z$. By the grid structure, each sub-rectangle can share boundary with at most $2 d$ other sub-rectangles.

Let $X_{1}$ be the union of all sub-rectangles that intersect $X$. Let $X_{2}$ be obtained from $X_{1}$ by adding all sub-rectangles that share boundary with it. Clearly,

$$
X \subseteq X_{1} \subseteq X_{2} \subseteq R
$$

By Lemma 3.3, we have that $p\left(X_{2}\right) \geqslant 2 d \cdot\left|X_{2}\right|^{(d-1) / d} \geqslant 2 d \cdot|X|^{(d-1) / d}$. Thus, in order to prove the first part of the lemma, it is enough to show that

$$
|E(X)| \geqslant|X|+5 p\left(X_{2}\right)
$$

First, let us show that

$$
\left|X_{2} \backslash X_{1}\right| \geqslant \frac{M_{1}}{2 d}\left(p^{R}\left(X_{1}\right)+p^{R}\left(X_{2}\right)\right) .
$$

Note that if $(\boldsymbol{n}, \boldsymbol{n}+\boldsymbol{e})$ is in $\partial^{R} X_{1}\left(\operatorname{resp} . \partial^{R}\left(R \backslash X_{2}\right)\right)$, then $\boldsymbol{n}+i \boldsymbol{e} \in X_{2} \backslash X_{1}$ for all $i \in\left[M_{1}\right]$. (Indeed, the directed edge $(\boldsymbol{n}, \boldsymbol{n}+\boldsymbol{e})$ enters some sub-rectangle $R^{\prime} \subseteq$ $X_{2} \backslash X_{1}$ and it takes at least $M_{1}$ steps in that direction before we leave $R^{\prime}$.) This way we encounter at least $M_{1} p^{R}\left(X_{1}\right)$ (resp. $M_{1} p^{R}\left(X_{2}\right)$ ) elements in $X_{2} \backslash X_{1}$ with each element counted at most $2 d$ times in total, which gives $(25)$.

Since we may assume that $2\left(M_{1}+1\right) \leqslant M$, each element of $X_{2}$ is within distance $M$ from $X$; thus $E(X) \supseteq \mathrm{B} \cap X_{2}$. Also, by the construction of $X_{1}$, we have $X \subseteq \mathrm{A} \cap X_{1}$. We conclude by (23) and (25) that

$$
\begin{aligned}
|E(X)|-|X| & \geqslant\left|\mathrm{B} \cap X_{2}\right|-\left|\mathrm{A} \cap X_{1}\right| \\
& \geqslant \delta\left|X_{2} \backslash X_{1}\right|-M_{0}\left(p\left(X_{1}\right)+p\left(X_{2}\right)\right) \\
& \geqslant \frac{\delta M_{1}}{2 d}\left(p^{R}\left(X_{1}\right)+p^{R}\left(X_{2}\right)\right)-M_{0}\left(p\left(X_{1}\right)+p\left(X_{2}\right)\right) .
\end{aligned}
$$

Let $i=1$ or 2 . Let us show that $p^{R}\left(X_{i}\right) \geqslant p\left(X_{i}\right) / M_{0}$. This directly follows from Lemma 4.4 if $\left|X_{i}\right| \leqslant \frac{2}{3}|R|$ since we can assume that $M_{0} \geqslant 9 d \rho$. So suppose that $\left|X_{i}\right|>\frac{2}{3}|R|$. Since $\mathrm{B} \cap X_{i} \subseteq E(X)$ has, by our assumption, less than $|\mathrm{B} \cap R| / 2$ elements, we have by (23) applied twice to the $\Phi$-uniform 
set B that

$$
\begin{aligned}
M_{0} p\left(X_{i}\right) & \geqslant \delta\left|X_{i}\right|-\left|\mathrm{B} \cap X_{i}\right| \geqslant \frac{2 \delta|R|}{3}-\frac{|\mathrm{B} \cap R|}{2} \\
& \geqslant \frac{2 \delta|R|}{3}-\frac{\delta|R|+M_{0} p(R)}{2}=\frac{\delta|R|}{6}-\frac{M_{0} p(R)}{2} .
\end{aligned}
$$

Since $|R| \geqslant(N / \rho)^{d}$ and $p(R) \leqslant 2 d N^{d-1}$ (and $N>M \gg M_{0}$ ), we conclude that

$$
p^{R}\left(X_{i}\right) \geqslant p\left(X_{i}\right)-p(R) \geqslant p\left(X_{i}\right) / 2,
$$

which is even stronger than claimed.

Now, by (26) and since $M_{1} \gg M_{0}$, we obtain the required bound (24):

$$
|E(X)|-|X| \geqslant\left(\frac{\delta M_{1}}{2 d M_{0}}-M_{0}\right)\left(p\left(X_{1}\right)+p\left(X_{2}\right)\right) \geqslant 5 p\left(X_{2}\right) .
$$

This finishes the proof of Part 1.

Now, we prove the second part of the lemma, assuming the validity of Part 1.

Let $\mathrm{A}_{0} \neq \emptyset$ consist of the unmatched points in $\mathrm{A} \cap R$. Define an alternating path as a sequence $\left(x_{0}, \ldots, x_{\ell}\right)$ such that $x_{0} \in \mathrm{A}_{0},\left(x_{i-1}, x_{i}\right) \in E \backslash \mathcal{M}$ for all odd $i \in[\ell]$ and $\left(x_{i}, x_{i-1}\right) \in \mathcal{M}$ for all even $i \in[\ell]$ (i.e. it is a path in $\mathcal{F}$ that starts with an unmatched vertex of $\mathrm{A} \cap R$ and alternates between unmatched and matched edges). Note that any alternating path that ends in an unmatched vertex is augmenting. For $i \in \mathbb{N}$, let $\mathrm{A}_{i}$ be the set of endpoints of alternating paths whose length is at most $i$ and has the same parity as $i$. (Thus $\mathrm{A}_{i} \subseteq \mathrm{A} \cap R$ for even $i$ and $\mathrm{A}_{i} \subseteq \mathrm{B} \cap R$ for odd $i$.) Let $\mathrm{A}_{i}^{\prime}$ consist of vertices reachable by an alternating path of length $i$ but not by a shorter one (that is, $\mathrm{A}_{i}^{\prime}:=\mathrm{A}_{i} \backslash \mathrm{A}_{i-2}$ for $i \geqslant 2$ and $\mathrm{A}_{i}^{\prime}:=\mathrm{A}_{i}$ for $i=0,1$ ).

Suppose on the contrary that there is no augmenting path of length at most $N$. Take an odd integer $\ell$ such that $N-3 \leqslant 2 \ell+1 \leqslant N$.

Roughly, the proof proceeds as follows. It is easy to see that $A_{2 i+1}=$ $E\left(\mathrm{~A}_{2 i}\right)$ for all $i \in \mathbb{N}$ and the absence of short augmenting paths implies that $\mathcal{M}$ covers all of $\mathrm{A}_{2 i+1}$ for $i \leqslant \ell$. It follows from Part 1 that $\left|\mathrm{A}_{i}\right|$ grows as $\Omega\left(i^{d}\right)$. By symmetry, the same applies when we grow alternating paths starting from B. These two processes have to collide in fewer than $N / 2$ steps, giving a contradiction. Let us provide the details now.

Clearly, if $i \leqslant \ell$, then $\mathrm{A}_{2 i+1}^{\prime} \subseteq \mathcal{M}(\mathrm{A})$ (i.e. every vertex of $\mathrm{A}_{2 i+1}^{\prime} \subseteq \mathrm{B} \cap R$ is matched) for otherwise we have a too short augmenting path. Furthermore, $\mathrm{A}_{2 i+2}^{\prime}=\mathcal{M}^{-1}\left(\mathrm{~A}_{2 i+1}^{\prime}\right)$ (i.e. $\mathcal{M}$ gives a bijection from $\mathrm{A}_{2 i+2}^{\prime}$ to $\left.\mathrm{A}_{2 i+1}^{\prime}\right)$. Thus, by induction on $i$, we have that

$$
\left|\mathrm{A}_{2 i+2}\right|=\left|\mathrm{A}_{2 i+1}\right|+\left|\mathrm{A}_{0}\right|, \quad \text { for every } i \leqslant \ell .
$$


Next, let us show that

$$
\left|\mathrm{A}_{\ell}\right| \geqslant|\mathrm{B} \cap R| / 2 .
$$

We may assume that $\left|\mathrm{A}_{i}\right|<|\mathrm{B} \cap R| / 2$ for all odd $i \leqslant \ell$, as otherwise we are done since $\mathrm{A}_{\ell} \supseteq \mathrm{A}_{i}$. Take any integer $i \in[0,(\ell-1) / 2]$. Since $\mathrm{A}_{2 i+1} \supseteq E\left(\mathrm{~A}_{2 i}\right)$ (in fact, this is equality), we have by Part 1 of the lemma that

$$
\left|\mathrm{A}_{2 i+1}\right| \geqslant\left|E\left(\mathrm{~A}_{2 i}\right)\right| \geqslant\left|\mathrm{A}_{2 i}\right|+10 d \cdot\left|\mathrm{A}_{2 i}\right|^{(d-1) / d} .
$$

If $i \geqslant 1$, then (27) implies that $\left|\mathrm{A}_{2 i}\right| \geqslant\left|\mathrm{A}_{2 i-1}\right|$. Thus the sizes $a_{i}:=\left|\mathrm{A}_{2 i-1}\right|$ satisfy $a_{1} \geqslant 1$ and

$$
a_{i+1} \geqslant a_{i}+10 d \cdot a_{i}^{(d-1) / d}, \quad \text { for all } i \in[(\ell-1) / 2] .
$$

Let an integer $\gamma=\gamma(d)$ be such that $\gamma^{j-1} \geqslant 5^{j-1}\left(\begin{array}{l}d \\ j\end{array}\right)$ for all $j \in[2, d]$; for example, we can take $\gamma=5 \cdot 2^{d}$. We obtain that $a_{i+1} \geqslant a_{i}+1$ and, if $a_{i} \geqslant c^{d}$ with $c \geqslant \gamma$ then we have by (29)

$a_{i+1} \geqslant c^{d}+10 d \cdot c^{d-1} \geqslant c^{d}+5 d \cdot c^{d-1}+\sum_{j=2}^{d} 5 c^{d-1} \geqslant \sum_{j=0}^{d}\left(\begin{array}{l}d \\ j\end{array}\right) 5^{j} c^{d-j}=(c+5)^{d}$.

We conclude by induction on $i$ that $a_{i+\gamma^{d}} \geqslant(5 i+\gamma)^{d}$ for every $i \in$ $\left[0,(\ell-1) / 2-\gamma^{d}\right]$. But then $a_{i}$ is larger than $N^{d} \geqslant|R|$ for some $i \leqslant N / 5+\gamma^{d} \leqslant$ $(\ell-1) / 2$. (Recall that $d \ll M<N \leqslant 2 \ell+4$.) This contradiction proves (28).

Likewise, by swapping the role of the sets $A$ and $B$ in the previous argument (and extending the definition of an alternating path accordingly), let $\mathrm{B}_{i}$ consist of vertices reachable by alternating paths that start in an unmatched vertex of $\mathrm{B} \cap R$ and whose length is at most $i$ and of the same parity as $i$. As above, we conclude that $\left|\mathrm{B}_{\ell}\right| \geqslant|\mathrm{A} \cap R| / 2$. Assume without loss of generality that $|\mathrm{B} \cap R| \geqslant|\mathrm{A} \cap R|$. By (27) and (28), we have that

$$
\left|\mathrm{A}_{\ell+1}\right|=\left|\mathrm{A}_{\ell}\right|+\left|\mathrm{A}_{0}\right|>\left|\mathrm{A}_{\ell}\right| \geqslant|\mathrm{B} \cap R| / 2 \geqslant|\mathrm{~A} \cap R| / 2 .
$$

Thus $\mathrm{B}_{\ell}$, which occupies at least half of $\mathrm{A} \cap R$, intersects $\mathrm{A}_{\ell+1}$.

Take two alternating paths $P:=\left(x_{0}, \ldots, x_{s}\right)$ and $P^{\prime}:=\left(y_{0}, \ldots, y_{t}\right)$ such that $x_{0} \in \mathrm{A}, y_{0} \in \mathrm{B}, x_{s}=y_{t}$, and $s+t$ is smallest possible; if there is more than one choice, let $s$ be maximum. Clearly, $s+t$ is odd and, by above, it is at most $2 \ell+1 \leqslant N$. Thus we cannot have $t=0$ for otherwise $P$ is a short augmenting path contradicting our assumption. Since $s$ was maximum, we cannot append $y_{t-1}$ to $P$ and still have an alternating path. By the parity of $s+t$, the only possible reason is that $y_{t-1}$ is equal to some $x_{j}$ with $s-j$ being a positive odd integer. But then $\left(x_{0}, \ldots, x_{j}\right)$ and $\left(y_{0}, \ldots, y_{t-1}\right)$ are alternating paths that contradict the choice of the pair $\left(P, P^{\prime}\right)$, namely, the minimality of $s+t$. This final contradiction proves Lemma 4.5. 


\section{Proof of Part 2 of Theorem 2.2}

In this section, let measurable mean Baire measurable.

Given the function $\Phi:\left\{2^{i}: i \in \mathbb{N}\right\} \rightarrow \mathbb{R}$, the vectors $\boldsymbol{x}_{1}, \ldots, \boldsymbol{x}_{d} \in \mathbb{T}^{k}$, and the sets $A, B \subseteq \mathbb{T}^{k}$ as in Part 2 of Theorem 2.2, let $M$ be sufficiently large and then let $r_{1}<r_{2}<\ldots$ be a fast-growing sequence of integers. (Namely, for each $i$, the constant $M$ and the subsequence $\left(r_{1}, \ldots, r_{i}\right)$ have to satisfy Lemma 5.2.)

Given our choice of $M$, consider the usual bipartite graph $\mathcal{G}=(A, B, E)$ as defined in (9); namely, $E=\left\{(\boldsymbol{a}, \boldsymbol{b}) \in A \times B: \boldsymbol{a}-\boldsymbol{b} \in \mathcal{V}_{M}\right\}$. Thus we would like to find a measurable perfect matching $\mathcal{M}$ in $\mathcal{G}$. We will use essentially the same construction that appears in Marks and Unger [26]. However, the analysis of its correctness for our problem is much more complicated.

Recall that a set $X \subseteq \mathbb{T}^{k}$ is called $r$-sparse if no $X_{\boldsymbol{u}}$ contains two distinct vectors at $L^{\infty}$-distance at most $r$. For every integer $i \geqslant 1$, choose Baire sets $A_{i} \subseteq A$ and $B_{i} \subseteq B$ such that $A_{i} \cap B_{i}=\emptyset, A_{i} \cup B_{i}$ is $\left(r_{i}+4 M\right)$-sparse and the sets $A^{\prime}:=A \backslash\left(\cup_{i=1}^{\infty} A_{i}\right)$ and $B^{\prime}:=B \backslash\left(\cup_{i=1}^{\infty} A_{i}\right)$ are meagre. For notational convenience, let us further agree that $A_{2 i+1}=B_{2 i}=\emptyset$ for all $i \in \mathbb{N}$, which will automatically take care of the disjointedness requirement. The existence of such sets is easy to establish. For example, let $\left\{\boldsymbol{y}_{i}: i \in \mathbb{N}\right\}$ be a dense subset of $\mathbb{T}^{k}$ and let $A_{2 i}$ (resp. $B_{2 i+1}$ ) be the intersection of $A$ (resp. $B$ ) with a ball centred at $\boldsymbol{y}_{i}$ of sufficiently small radius (namely, so that the ball is $\left(r_{2 i+1}+4 M\right)$-sparse). The closure of $A^{\prime} \cup B^{\prime}$ cannot contain a non-empty open set $U$ because it avoids a ball around some $\boldsymbol{y}_{i} \in U$. Thus $A^{\prime} \cup B^{\prime}$ is in fact nowhere dense.

In order to prove Theorem 2.2, it is enough to find measurable nested matchings $\mathcal{M}_{1} \subseteq \mathcal{M}_{2} \subseteq \ldots$ such that for all $i \geqslant 1$ we have

$$
\mathcal{M}_{i}^{-1}(B) \supseteq A_{i} \quad \text { and } \quad \mathcal{M}_{i}(A) \supseteq B_{i} .
$$

Indeed, suppose that such matchings $\mathcal{M}_{i}$ exist. Let $\mathcal{M}:=\cup_{i=1}^{\infty} \mathcal{M}_{i}$. Let $X \subseteq$ $\mathbb{T}^{k}$ consist of the translates of the meagre set $A^{\prime} \cup B^{\prime}$ by integer combinations of $\boldsymbol{x}_{1}, \ldots, \boldsymbol{x}_{d}$. Then $X$ is a meagre invariant set and the restriction of $\mathcal{M}$ to $\mathcal{G}[A \backslash X, B \backslash X]$ is a perfect matching by (30). If we replace the restriction of the injection $\mathcal{M}: A \rightarrow B$ to $A \cap X$ by the one provided by Theorem 2.1 (with respect to the same vectors $\boldsymbol{x}_{1}, \ldots, \boldsymbol{x}_{d}$ ), then we obtain the required measurable perfect matching in $\mathcal{G}$.

The following lemma is a special case of the inductive step in Marks and Unger [26], slightly adopted to our purposes. A matching $\mathcal{M}$ in a graph $G$ is called $G$-extendable (or extendable when $G$ is understood) if $G$ has a (not necessarily measurable) perfect matching $\mathcal{M}^{\prime} \supseteq \mathcal{M}$. 
LEMma 5.1. Under the assumptions of this section, for every $i \geqslant 1$ and every measurable $\mathcal{G}$-extendable matching $\mathcal{M}_{i-1}$, there is a measurable matching $\mathcal{M}_{i} \supseteq \mathcal{M}_{i-1}$ satisfying (30) and the following properties.

(1) The added edge-set is $\left(r_{i}+2 M\right)$-sparse, that is, for every $\boldsymbol{u} \in \mathbb{T}^{k}$ and distinct $(\boldsymbol{a}, \boldsymbol{b}),(\boldsymbol{x}, \boldsymbol{y}) \in\left(\mathcal{M}_{i} \backslash \mathcal{M}_{i-1}\right)_{\boldsymbol{u}}$, the distance between the sets $\{\boldsymbol{a}, \boldsymbol{b}\}$ and $\{\boldsymbol{x}, \boldsymbol{y}\}$ (as defined in (11)) is larger than $r_{i}+2 M$.

(2) $\mathcal{M}_{i} \backslash \mathcal{M}_{i-1} \subseteq \mathcal{M}_{i-1}^{\equiv}$, where $\mathcal{M}_{i-1}^{\equiv}$ consists of those $(\boldsymbol{a}, \boldsymbol{b}) \in E \backslash \mathcal{M}_{i-1}$ such that $\mathcal{M}_{i-1} \cup\{(\boldsymbol{a}, \boldsymbol{b})\}$ is a $\mathcal{G}$-extendable matching.

Proof. By the symmetry between $A$ and $B$, assume that, for example, $i$ is even. Since $B_{i}=\emptyset$, we just need to match every vertex of $X:=A_{i} \backslash \mathcal{M}_{i-1}^{-1}(B)$ in order to satisfy (30). By the measurability of $\mathcal{M}_{i-1}$, the set $X \subseteq \mathbb{T}^{k}$ is Baire.

For $\boldsymbol{x} \in X$, let $Y(\boldsymbol{x}):=\left\{\boldsymbol{y} \in E(\boldsymbol{x}):(\boldsymbol{x}, \boldsymbol{y}) \in \mathcal{M}_{\overline{i-1}}^{\bar{\equiv}}\right\}$ consist of those neighbours $\boldsymbol{y}$ of $\boldsymbol{x}$ for which $\mathcal{M}_{i-1} \cup\{(\boldsymbol{x}, \boldsymbol{y})\}$ is an extendable matching. This set is non-empty by the assumed extendability of $\mathcal{M}_{i-1}$. Furthermore, for $j \in \mathbb{N}$, let $Y_{j}(\boldsymbol{x})$ consist of those $\boldsymbol{y} \in E(\boldsymbol{x})$ such that $\mathcal{M}_{i-1} \cup\{(\boldsymbol{x}, \boldsymbol{y})\}$ can be extended to a matching that covers all vertices of $\mathcal{G}$ (in both parts) at the coset distance at most $j$ from $\boldsymbol{x}$; namely we require that $\mathcal{G}_{\boldsymbol{x}}$ has a matching $\mathcal{M} \supseteq \mathcal{M}_{i-1, \boldsymbol{x}}$ such that

$$
\mathcal{M}\left(A_{\boldsymbol{x}}\right) \supseteq \operatorname{dist}_{\leqslant j}(\mathbf{0}) \cap B_{\boldsymbol{x}} \quad \text { and } \quad \mathcal{M}^{-1}\left(B_{\boldsymbol{x}}\right) \supseteq \operatorname{dist}_{\leqslant j}(\mathbf{0}) \cap A_{\boldsymbol{x}} .
$$

Clearly, $Y(\boldsymbol{x}) \subseteq \cap_{j \in \mathbb{N}} Y_{j}(\boldsymbol{x})$. The converse inclusion holds by the Compactness Principle (or a direct diagonalisation argument) applied to the locally finite graph $\mathcal{G}_{\boldsymbol{x}}$. Thus $Y(\boldsymbol{x})=\cap_{j \in \mathbb{N}} Y_{j}(\boldsymbol{x})$.

Fix some Borel map $\chi: \mathbb{T}^{k} \rightarrow[t]$ with $2 M$-sparse pre-images provided by Lemma 3.2. Let $\mathcal{M}_{i}$ be obtained from $\mathcal{M}_{i-1}$ by adding, for each $\boldsymbol{x} \in X$, the pair $(\boldsymbol{x}, \boldsymbol{y})$, where $\boldsymbol{y}$ is the element of $Y(\boldsymbol{x}) \neq \emptyset$ with the smallest value of $\chi$. Since $X \subseteq A_{i}$ is $\left(r_{i}+4 M\right)$-sparse, $\mathcal{M}_{i}$ satisfies Part 1 (in particular, $\mathcal{M}_{i}$ is a matching). Clearly, Part 2 and (30) hold by the definition of $\mathcal{M}_{i}$.

Thus it remains to verify the measurability of $\mathcal{M}_{i}$. To this end, it is enough to prove that for every $\boldsymbol{v} \in \mathcal{V}_{M}$ the set $Z_{\boldsymbol{v}}:=\left\{\boldsymbol{x} \in X:(\boldsymbol{x}, \boldsymbol{x}+\boldsymbol{v}) \in \mathcal{M}_{i} \backslash \mathcal{M}_{i-1}\right\}$ is measurable. Trivially, $Z_{\boldsymbol{v}}$ is the union over $m \in[t]$ of

$$
Z_{\boldsymbol{v}, m}:=\left\{\boldsymbol{x} \in Z_{\boldsymbol{v}}: \chi(\boldsymbol{x}+\boldsymbol{v})=m\right\} .
$$

We prove by induction on $m=1, \ldots, t$ that, for each $\boldsymbol{v} \in \mathcal{V}_{M}$, the set $Z_{\boldsymbol{v}, m}$ is measurable (which will finish the proof of the lemma). For the base case, note that $Z_{\boldsymbol{v}, 1}$ consists exactly of those $\boldsymbol{x}$ in the translated Borel set $\chi^{-1}(1)-\boldsymbol{v}$ such that for every radius $j$ there exists $\mathcal{M} \supseteq \mathcal{M}_{i-1, \boldsymbol{x}}$ satisfying (31). The latter property, for any given $j$, is clearly determined by the picture inside the ball $\operatorname{dist}_{\leqslant j+M}(\mathbf{0})$ in the coset of $\boldsymbol{x}$ and can be checked by a $(j+M)$-local rule. Thus each $Z_{\boldsymbol{v}, 1}$ is measurable by Lemma 3.1. The measurability of $Z_{\boldsymbol{v}, m}$ for 
$m \geqslant 2$ follows by induction: the formula for $Z_{\boldsymbol{v}, m}$ is the trivial adaptation of that for $Z_{\boldsymbol{v}, 1}$ except we additionally have to exclude $\cup_{q=1}^{m-1} \cup_{\boldsymbol{w} \in \mathcal{V}_{M}} Z_{\boldsymbol{w}, q}$, the set of vertices $\boldsymbol{x} \in X$ for which $Y(\boldsymbol{x})$ has an element whose $\chi$-value is smaller than $m$. (We do not need to worry that the matches of $X$ in $B$ may collide as they are automatically distinct by the $2 M$-sparseness of $X$.)

Armed with Lemma 5.1, we can now describe how we construct the desired matchings. We start with the empty matching $\mathcal{M}_{0}$ (which is $\mathcal{G}$-extendable by Theorem 2.1) and try to iteratively apply Lemma 5.1 for $i=1,2, \ldots$, constructing nested measurable matchings $\mathcal{M}_{1} \subseteq \mathcal{M}_{2} \subseteq \ldots$ in $\mathcal{G}$ that satisfy (30). If each new matching $\mathcal{M}_{i}$ is extendable, then Lemma 5.1 can always be applied, giving the proof of Theorem 2.2 as discussed above.

The extendability of each $\mathcal{M}_{i}$ directly follows from Lemma 5.2 below (when applied to the $\Phi$-uniform sets $\mathrm{A}:=A_{\boldsymbol{u}}$ and $\mathrm{B}:=B_{\boldsymbol{u}}$ for each $\boldsymbol{u} \in \mathbb{T}^{k}$ ) since, clearly, it is enough to verify extendability inside each coset. Lemma 5.2 (like Lemma 4.5) is a purely combinatorial statement with a rather long proof.

Lemma 5.2. For every integer $d \geqslant 1$, real $\delta>0$, and function $\Phi$ : $\left\{2^{i}: i \in \mathbb{N}\right\} \rightarrow \mathbb{R}$ satisfying $\sum_{i=0}^{\infty} \Phi\left(2^{i}\right) / 2^{(d-1) i}<\infty$, there is $M=M(d, \delta, \Phi)$ such that the following holds.

Let $\mathrm{A}, \mathrm{B} \subseteq \mathbb{Z}^{d}$ be $\Phi$-uniform sets of density $\delta>0$. Let positive integers $r_{1} \leqslant \ldots \leqslant r_{i}$ satisfy

$$
\sum_{j=1}^{i}\left(M / r_{j}\right)^{(d-1) / d} \leqslant 4^{1-d} .
$$

Let $\mathcal{M}_{0}:=\emptyset \subseteq \mathcal{M}_{1} \subseteq \ldots \subseteq \mathcal{M}_{i}$ be matchings in the bipartite graph

$$
\mathcal{F}:=\left(\mathrm{A}, \mathrm{B},\left\{(\boldsymbol{a}, \boldsymbol{b}) \in \mathrm{A} \times \mathrm{B}:\|\boldsymbol{a}-\boldsymbol{b}\|_{\infty} \leqslant M\right\}\right)
$$

such that $\mathcal{N}_{j}:=\mathcal{M}_{j} \backslash \mathcal{M}_{j-1}$ is $\left(r_{j}+2 M\right)$-sparse for each $j \in[i], \mathcal{M}_{i} \neq \emptyset$, and $\mathcal{M}_{i-1} \cup\{(\boldsymbol{a}, \boldsymbol{b})\}$ is $\mathcal{F}$-extendable for every $(\boldsymbol{a}, \boldsymbol{b}) \in \mathcal{M}_{i}$. Then $\mathcal{M}_{i}$ is $\mathcal{F}$ extendable.

Proof. Given $d, \delta$, and $\Phi$ as above, let $M_{0} \ll M$ be sufficiently large integers. For convenience, assume that $M$ is a power of 2 . By our assumption on $\Phi$, we can also require that $\Phi(M / 2)<\delta(M / 2)^{d}$. As in the proof of Lemma 4.5, assume that $M_{0}$ satisfies (23).

For $\ell \in \mathbb{N}$ and $X \subseteq \mathbb{Z}^{d}$, we say that $\boldsymbol{y}, \boldsymbol{z} \in \mathbb{Z}^{d}$ are $(\ell, X)$-connected if we can find a (possibly empty) sequence $\boldsymbol{x}_{1}, \ldots, \boldsymbol{x}_{n}$ of elements of $X$ such that, letting $\boldsymbol{x}_{0}:=\boldsymbol{y}$ and $\boldsymbol{x}_{n+1}:=\boldsymbol{z}$, we have that $\operatorname{dist}\left(\boldsymbol{x}_{j-1}, \boldsymbol{x}_{j}\right) \leqslant \ell$ for all $j \in[n+1]$. In other words, we can travel from $\boldsymbol{y}$ to $\boldsymbol{z}$ via $X$ using $\ell$-jumps (i.e. steps of $L^{\infty}$-distance at most $\ell$ ). The set $X$ is $\ell$-connected if every $\boldsymbol{x}, \boldsymbol{x}^{\prime} \in$ $X$ are $(\ell, X)$-connected. The binary relation of being $(\ell, X)$-connected when restricted to $X$ is clearly an equivalence relation. Its equivalence classes will 
be called $\ell$-components of $X$. Equivalently, an $\ell$-component is just a maximal $\ell$-connected subset of $X$.

For $j \in[0, i]$, we let $\mathrm{A}_{j}:=\mathrm{A} \backslash \mathcal{M}_{j}^{-1}(\mathrm{~B})$ and $\mathrm{B}_{j}:=\mathrm{B} \backslash \mathcal{M}_{j}(\mathrm{~A})$; also, for a subset $Y$ of $\mathrm{A}_{j}$ or $\mathrm{B}_{j}$, let $\Gamma_{j}(Y)$ denote the set of its neighbours with respect to the induced bipartite subgraph

$$
\mathcal{F}_{j}:=\mathcal{F}\left[\mathrm{A}_{j}, \mathrm{~B}_{j}\right]=\left(\mathrm{A}_{j}, \mathrm{~B}_{j},\left\{(\boldsymbol{a}, \boldsymbol{b}) \in \mathrm{A}_{j} \times \mathrm{B}_{j}:\|\boldsymbol{a}-\boldsymbol{b}\|_{\infty} \leqslant M\right\}\right),
$$

which is obtained from $\mathcal{F}$ by removing the vertices matched by $\mathcal{M}_{j}$.

Clearly, the matching $\mathcal{M}_{i}$ is $\mathcal{F}$-extendable if and only if $\mathcal{F}_{i}$ has a perfect matching. Since $\mathcal{F}_{i}$ is locally finite, Rado's theorem [30] applies. Hence, it is enough to show that (8) holds for $\mathcal{F}_{i}$.

Since $2 M$-components of any set are at distance larger than $2 M$ from each other, their neighbourhoods in $\mathcal{F}_{i}$ are disjoint; thus it is enough to prove that

$$
\left|\Gamma_{i}(X)\right| \geqslant|X|, \quad \text { for every finite } 2 M \text {-connected } X \subseteq \mathrm{A}_{i} \text { or } \mathrm{B}_{i} .
$$

So take an arbitrary non-empty finite $2 M$-connected set $X$ in one part, say $X \subseteq \mathrm{A}_{i}$.

Given $X$, let its reference point be the vector $\boldsymbol{o}(X) \in \mathbb{Z}^{d}$ whose $j$-th coordinate for $j \in[d]$ is the minimum of the $j$-th coordinate projection $\operatorname{Pr}_{j}$ : $X \rightarrow \mathbb{Z}$. Partition $\mathbb{Z}^{d}$ into the $(M / 2)$-regular grid $\mathcal{Q}$ with $\boldsymbol{o}(X)$ as the origin. Let $X_{1} \subseteq \mathbb{Z}^{d}$ be the union of all cubes in $\mathcal{Q}$ that intersect $X$ and let $X_{2} \supseteq X_{1}$ be obtained from $X_{1}$ by adding cubes from $\mathcal{Q}$ that share boundary with $X_{1}$. This definition is a special case of the one in the proof of Lemma 4.5 if we let $M_{1}:=M / 2$ and take a rectangle $R \supseteq \operatorname{dist}_{\leqslant M+1}(X)$ aligned with the $M_{1^{-}}$ regular grid centred at $\boldsymbol{o}(X)$. In particular, the proof of (26) from Lemma 4.5 applies verbatim to the current definitions of $X_{1}$ and $X_{2}\left(\right.$ with $p^{R}(Y)=p(Y)$ for $Y \subseteq X_{2}$ ) and gives that, for example,

$$
\left|\Gamma_{0}(X)\right|-|X| \geqslant\left|\mathrm{B} \cap X_{2}\right|-\left|\mathrm{A} \cap X_{1}\right| \geqslant p\left(X_{1}\right) .
$$

By a hole $($ of $X)$ we will mean a $2 M$-component of $\mathbb{Z}^{d} \backslash X_{1}$. In other words, two vertices of $\mathbb{Z}^{d} \backslash X_{1}$ are in the same hole if and only if one can travel from one to the other in $2 M$-jumps staying all the time in $\mathbb{Z}^{d} \backslash X_{1}$. Let $\mathcal{H}(X)$ be the set of all holes of $X$, including the (unique) infinite one, which we denote by $H_{\infty}$. Note that the boundary of $X_{1}$ is the disjoint union of the (reversed) boundaries of the holes. Call a hole $H \in \mathcal{H}(X)$ rich if $p(H) \geqslant\left(r_{i} / M\right)^{(d-1) / d}$.

Rather roughly, the main ideas behind the proof are as follows. If no edge of $\mathcal{N}_{i}$ is within distance $M$ from $X$, then $\Gamma_{i}(X)=\Gamma_{i-1}(X)$ and (33) holds since $\mathcal{M}_{i-1}$ is extendable by the assumption of the lemma. If there is only one $(\boldsymbol{a}, \boldsymbol{b}) \in \mathcal{N}_{i}$ close to $X$, then (33) still holds because $\mathcal{M}_{i}$ near $X$ is the same as the extendable matching $\mathcal{M}_{i-1} \cup\{(\boldsymbol{a}, \boldsymbol{b})\}$. Thus a problem can only arise if at least two edges of $\mathcal{N}_{i}$ are $M$-close to $X$. The $\left(r_{i}+2 M\right)$-sparseness of $\mathcal{N}_{i}$ and the $2 M$-connectivity of $X$ imply that $|X| \geqslant r_{i} / 2 M$. Thus these assumptions (that 
follow from the construction of Lemma 5.1) automatically take care of (33) when the set $X$ is "small". This simple and yet beautiful idea is from Marks and Unger [26]. It worked well in their setting when their initial assumption was that $\left|\Gamma_{0}(X)\right| \geqslant(1+\varepsilon)|X|$ for some absolute constant $\varepsilon>0$. Our graph $\mathcal{F}_{0}$ does not have such strong expansion property (since the group of translations is amenable) but it satisfies (34). When we pass to $\mathcal{F}_{i}$ by removing the sparse matchings $\mathcal{N}_{1}, \ldots, \mathcal{N}_{i}$ from $\mathcal{F}_{0}$, only those removed edges that are near to $\partial X_{1}$ can decrease the value of $\left|\Gamma_{i}(X)\right|-|X|$ when compared to $\left|\Gamma_{0}(X)\right|-|X|$. If $H \in \mathcal{H}(X)$ is a hole, then we expect that at most $O\left(p(H) / r_{j}+1\right)$ edges of $\mathcal{N}_{j}$ can come near $\partial H$ for each $j \in[i]$. Thus, the "loss" from the hole should be at most $\sum_{j=1}^{i} O\left(p(H) / r_{j}+1\right)$. This is smaller than $p(H)$, the hole's contribution to $p\left(X_{1}\right)$, if $p(H)$ is sufficiently large. Thus, rich holes should only help us. So, the remaining problematic case is when $|X|$ is relatively large but contain at least one hole $H$ which is not rich. Here we take non-rich finite holes $H \in \mathcal{H}(X)$ one by one. For each such $H$, we "fill" it up; namely, we decrease the matchings to avoid $H$ and enlarge $X$ so that $H$ disappears from $\mathcal{H}(X)$. As we will see, this operation does not increase $\left|\Gamma_{i}(X)\right|-|X|$. By iterating it, we can get rid of all non-rich finite holes. Now, if we can prove (33) for the final set, then the original set $X$ also satisfies this inequality.

Let us provide the details of the proof.

Case 1 At most one edge $(\boldsymbol{a}, \boldsymbol{b})$ of $\mathcal{N}_{i}=\mathcal{M}_{i} \backslash \mathcal{M}_{i-1}$ satisfies $\operatorname{dist}(\{\boldsymbol{a}, \boldsymbol{b}\}, X) \leqslant M$.

Suppose that such an edge $(\boldsymbol{a}, \boldsymbol{b})$ exists, for otherwise $\Gamma_{i}(X)=\Gamma_{i-1}(X)$ has at least $|X|$ vertices (since $\mathcal{M}_{i-1}$ is $\mathcal{F}$-extendable), as required. Let $\mathcal{M}_{\infty}$ be some perfect matching of $\mathcal{F}_{0}=\mathcal{F}$ that extends $\mathcal{M}_{i-1} \cup\{(\boldsymbol{a}, \boldsymbol{b})\}$. The matching $\mathcal{M}_{\infty}$ gives an injection from $X$ to $\Gamma_{i-1}(X) \backslash\{\boldsymbol{b}\} \subseteq \Gamma_{i}(X)$. (Note that $\boldsymbol{a} \in \mathcal{M}_{i}^{-1}(\mathrm{~B})$ cannot belong to $X \subseteq \mathrm{A}_{i}$.) Thus (33) holds.

Case 2 We are not in Case 1, that is, at least two edges of $\mathcal{N}_{i}$ are within distance $M$ from $X$.

Fix some $e \neq e^{\prime}$ in $\mathcal{N}_{i}$ as above. By the $2 M$-connectedness of $X$, we can connect $e$ to $e^{\prime}$ by jumps of distance at most $2 M$ with all intermediate vertices lying in $X$. This means that $|X| \geqslant \operatorname{dist}\left(e, e^{\prime}\right) / 2 M-1 \geqslant r_{i} / 2 M$. Consider the boundary $\partial H_{\infty}$ of the infinite hole $H_{\infty} \in \mathcal{H}(X)$. The set $X^{\prime}:=\mathbb{Z}^{d} \backslash H_{\infty} \supseteq X$ contains at least $r_{i} / 2 M$ elements. Also, $X^{\prime}$ is finite because, for example, it lies in the convex hull of the finite set $X_{1}$. By the isoperimetric inequality of Lemma 3.3, we have

$$
p\left(H_{\infty}\right)=p\left(X^{\prime}\right) \geqslant 2 d \cdot\left|X^{\prime}\right|^{(d-1) / d} \geqslant\left(r_{i} / M\right)^{(d-1) / d},
$$

so $H_{\infty}$ is a rich hole.

Case 2.1 Every hole $H \in \mathcal{H}(X)$ is rich. 
Note that every cube $Q \subseteq X_{2}$ from the $\boldsymbol{o}(X)$-centred $(M / 2)$-grid is inside $X_{1}$ or touches some $(M / 2)$-cube of $X_{1}$. Since each grid cube of $X_{1}$ contains a vertex from $X$, we have that $\Gamma_{0}(X) \supseteq \mathrm{B} \cap X_{2}$. When we pass to $\Gamma_{i}(X)$, all vertices of $\mathrm{B} \cap X_{2}$ that are not matched by $\mathcal{M}_{i}$ remain. Thus $\Gamma_{i}(X) \supseteq\left(\mathrm{B} \cap X_{2}\right) \backslash \mathcal{M}_{i}(\mathrm{~A})$. Since $X \subseteq \mathrm{A}_{i}$ does not contain any $\mathcal{M}_{i}$-matched vertex, we also have $X \subseteq$ $\left(\mathrm{A} \cap X_{1}\right) \backslash \mathcal{M}_{i}^{-1}(\mathrm{~B})$. We conclude that

$$
\left|\Gamma_{i}(X)\right|-|X| \geqslant\left|\mathrm{B} \cap X_{2}\right|-\left|\mathrm{A} \cap X_{1}\right|-\lambda,
$$

where $\lambda:=\left|\mathcal{M}_{i}(\mathrm{~A}) \cap X_{2}\right|-\left|\mathcal{M}_{i}^{-1}(\mathrm{~B}) \cap X_{1}\right|$. In view of (34), it suffices to show that $\lambda \leqslant p\left(X_{1}\right)$.

Fix any $j \in[i]$. Let $\Lambda_{j}$ consist of those $e$ in $\mathcal{N}_{j}=\mathcal{M}_{j} \backslash \mathcal{M}_{j-1}$ that contribute a positive amount (that is, +1 ) to $\lambda$. For $e \in \Lambda_{j}$ let its private set be

$$
\mathcal{P}_{j}(e):=\left\{e^{\prime} \in \partial X_{1}: \operatorname{dist}\left(e, e^{\prime}\right) \leqslant r_{j} / 2+M\right\} .
$$

Let us show that each private set is relatively large:

Claim 5.2.1. For every $e \in \Lambda_{j}$, we have $\left|\mathcal{P}_{j}(e)\right| \geqslant\left(r_{j} / M\right)^{(d-1) / d}$.

Proof of Claim. Let $e=(\boldsymbol{a}, \boldsymbol{b})$. Since $e$ contributes +1 to $\lambda$, we have that $\boldsymbol{b} \in X_{2}$ and $\boldsymbol{a} \notin X_{1}$. Let $H \in \mathcal{H}(X)$ be the hole which contains $\boldsymbol{a}$. Suppose that $\partial H \backslash \mathcal{P}_{j}(e) \neq \emptyset$, for otherwise we are done since $r_{j} \leqslant r_{i}$ and every hole is rich.

Let us show that, for every integer $m$ with $0 \leqslant m \leqslant r_{j} / 4 M$, the annulus

$$
\mathcal{O}_{m}:=\left\{\boldsymbol{n} \in \mathbb{Z}^{d}:(2 m-1) M<\operatorname{dist}(\boldsymbol{n}, e) \leqslant(2 m+1) M\right\}
$$

contains at least one element of $\partial H$ as a subset. The inner part $\cup_{t=0}^{m} \mathcal{O}_{t}$ of $\mathcal{O}_{m}$ contains elements from both $H$ (namely, $\boldsymbol{a}$ ) and $X$ (namely, all elements of the set $\operatorname{dist}_{\leqslant M}(\boldsymbol{b}) \cap X$ which is non-empty since $\left.\boldsymbol{b} \in X_{2}\right)$. The same holds for the outer part $\cup_{t=m+1}^{\infty} \mathcal{O}_{t}$ of $\mathcal{O}_{m}$ because it entirely contains every edge of $\partial H \backslash \mathcal{P}_{j}(e) \neq \emptyset$. Each of the sets $H$ and $X$ is $2 M$-connected; thus we can travel within the set from the inner to the outer part of $\mathcal{O}_{m}$ in $2 M$-jumps. The distance to $e$ changes by at most $2 M$ at each step, so there must be a moment when we land in the annulus $\mathcal{O}_{m}$. Thus $\mathcal{O}_{m}$ contains elements from both $H$ and $X$. It is easy to see that one can travel within $\mathcal{O}_{m}$ using only steps of $L^{1}$-distance 1 between its any two vertices, in particular, from $\mathcal{O}_{m} \cap H$ to $\mathcal{O}_{m} \cap X$. Since $H \cap X=\emptyset$, there is a step from $H$ to its complement, giving the required element of $\partial H$ inside $\mathcal{O}_{m}$.

Since the annuli are disjoint for different $m$, this gives at least $r_{j} / 4 M$ different elements of $\partial H$, all belonging to $\mathcal{P}_{j}(e)$. This is at least the desired bound on $\left|\mathcal{P}_{j}(e)\right|$ since $r_{j} / M \geqslant 4^{d}$ by (32). The claim is proved.

Since $\mathcal{N}_{j}$ is $\left(r_{j}+2 M\right)$-sparse by the assumptions of the lemma, we have that $\mathcal{P}_{j}(e) \cap \mathcal{P}_{j}\left(e^{\prime}\right)=\emptyset$ for all distinct $e, e^{\prime} \in \Lambda_{j}$. Thus Claim 5.2.1 implies that 
$\left|\Lambda_{j}\right| \leqslant p\left(X_{1}\right) /\left(r_{j} / M\right)^{(d-1) / d}$. Since $j \in[i]$ was arbitrary, we conclude by (32) that

$$
\lambda \leqslant \sum_{j=1}^{i}\left|\Lambda_{j}\right| \leqslant p\left(X_{1}\right) \sum_{j=1}^{i}\left(M / r_{j}\right)^{(d-1) / d} \leqslant p\left(X_{1}\right) .
$$

Thus we derive from (34) and (35) that $\left|\Gamma_{i}(X)\right| \geqslant|X|$, as required.

Case 2.2 We are not in Case 2.1, that is, there is at least one non-rich hole in $\mathcal{H}(X)$.

Take a hole $H \in \mathcal{H}(X)$ which is not rich. We have $H \neq H_{\infty}$ because, as we argued at the beginning of Case 2, the infinite hole is necessarily rich.

We claim that at most one edge $(\boldsymbol{a}, \boldsymbol{b}) \in \mathcal{N}_{i}$ satisfies $\boldsymbol{a} \in \operatorname{dist}_{\leqslant M}(H)$. Indeed, if $(\boldsymbol{a}, \boldsymbol{b}),\left(\boldsymbol{a}^{\prime}, \boldsymbol{b}^{\prime}\right) \in \mathcal{N}_{i}$ contradict this, then we can connect $\boldsymbol{a}$ to $\boldsymbol{a}^{\prime}$ by using jumps of distance at most $2 M$ with all intermediate vertices belonging to the hole $H$. This gives at least $r_{i} / 2 M$ vertices in $H$, contradicting by Lemma 3.3 our assumption that the hole $H$ is not rich.

If it exists, let $e:=(\boldsymbol{a}, \boldsymbol{b})$ be the unique edge of $\mathcal{N}_{i}$ with $\boldsymbol{a} \in \operatorname{dist}_{\leqslant M}(H)$. By our assumptions, there is a perfect matching $\mathcal{M}_{\infty}$ in $\mathcal{F}_{0}$ such that $\mathcal{M}_{\infty} \supseteq$ $\mathcal{M}_{i-1}$ and, if $e$ exists, then $\mathcal{M}_{\infty}$ also contains $e$. For $j \in[0, i]$, define

$$
\mathcal{M}_{j}^{\prime}:=\left\{(\boldsymbol{x}, \boldsymbol{y}) \in \mathcal{M}_{j}: \operatorname{dist}(\boldsymbol{x}, H)>M\right\},
$$

and let $\Gamma_{j}^{\prime}$ denote the neighbourhood taken with respect to the graph

$$
\mathcal{F}_{j}^{\prime}:=\mathcal{F}\left[\mathrm{A} \backslash\left(\mathcal{M}_{j}^{\prime}\right)^{-1}(\mathrm{~B}), \mathrm{B} \backslash \mathcal{M}_{j}^{\prime}(\mathrm{A})\right]
$$

which is obtained from $\mathcal{F}$ by removing all vertices matched by $\mathcal{M}_{j}^{\prime}$. Also, define

$$
X^{\prime}:=X \cup\left(\mathrm{A} \cap \operatorname{dist}_{\leqslant M}(H)\right) .
$$

The following claim will allow us to pass from $X$ to $X^{\prime}$ when proving (33).

Claim 5.2.2. All of the following properties hold.

(1) The sets $X$ and $X^{\prime}$ have the same reference points, that is, $\boldsymbol{o}\left(X^{\prime}\right)=$ $\boldsymbol{o}(X)$.

(2) The set $X_{1}^{\prime}$ (which consists of all cubes from the $(M / 2)$-grid centred at $\boldsymbol{o}\left(X^{\prime}\right)$ that intersect $X^{\prime}$ ) equals $X_{1} \cup H$.

(3) When we pass to $X^{\prime}$, the hole $H$ disappears; specifically, $\mathcal{H}\left(X^{\prime}\right)=$ $\mathcal{H}(X) \backslash\{H\}$.

(4) The set $X^{\prime}$ is $2 M$-connected.

(5) We have that $\left|\Gamma_{i}(X)\right|-|X| \geqslant\left|\Gamma_{i}^{\prime}\left(X^{\prime}\right)\right|-\left|X^{\prime}\right|$.

Proof of Claim. Suppose that the first property does not hold, say $X^{\prime}$ contains some $\boldsymbol{n}$ whose $j$-th coordinate is strictly smaller than $\min \left(\operatorname{Pr}_{j}(X)\right)$. But then $\boldsymbol{n} \in \mathbb{Z}^{d} \backslash X$ belongs to the infinite hole $H_{\infty}$ as demonstrated by the infinite path with vertices $\boldsymbol{n}-m \boldsymbol{e}_{j}$ for $m \in \mathbb{N}$. By the definition of $X^{\prime}$, we 
have $\operatorname{dist}(\boldsymbol{n}, H) \leqslant M$ and thus $\operatorname{dist}\left(H, H_{\infty}\right) \leqslant M$, contradicting $H \neq H_{\infty}$. This proves Part 1.

Let us turn to Part 2. Since $X^{\prime} \supseteq X$ and $\boldsymbol{o}\left(X^{\prime}\right)=\boldsymbol{o}(X)$, we have that $X_{1}^{\prime} \supseteq X_{1}$. Recall that, when we were choosing $M$, one of the required properties was that $\Phi(M / 2)<\delta(M / 2)^{d}$. This implies that every $(M / 2)$-cube intersects the $\Phi$-uniform set A. Thus any $(M / 2)$-cube $Q \subseteq H$ contains at least one element of $\mathrm{A} \cap H \subseteq X^{\prime} \backslash X$, which implies that $X_{1}^{\prime} \supseteq H$. On the other hand, every other hole $H^{\prime} \in \mathcal{H}(X) \backslash\{H\}$ is at distance at least $M$ from $X^{\prime} \backslash X \subseteq \operatorname{dist}_{\leqslant M}(H)$, so none of the vertices of $H^{\prime}$ can be claimed when we build $X_{1}^{\prime}$. This proves Part 2 .

Part 3 follows directly from Part 2.

We know that every $(M / 2)$-cube intersects $\mathrm{A}$, so every element of $\mathrm{A} \cap H$ can be connected to $X$ using $M$-jumps with all intermediate points in $\mathrm{A} \cap H$. This, the $2 M$-connectivity of $X$, and Part 2 imply Part 4 .

It remains to verify the last part. Since $X^{\prime} \supseteq X$ and $\mathcal{F}_{i}^{\prime} \supseteq \mathcal{F}_{i}$, we have that $\Gamma_{i}^{\prime}\left(X^{\prime}\right) \supseteq \Gamma_{i}(X)$. Thus we can equivalently rewrite Part 5 as $\left|X^{\prime} \backslash X\right| \geqslant|W|$, where $W:=\Gamma_{i}^{\prime}\left(X^{\prime}\right) \backslash \Gamma_{i}(X)$. Hence, it suffices to show that the injection $\mathcal{M}_{\infty}^{-1}$ maps $W$ into $X^{\prime} \backslash X$. Take any $\boldsymbol{w} \in W$ and let $\boldsymbol{v}:=\mathcal{M}_{\infty}^{-1}(\boldsymbol{w}) \in A$. Thus we aim at showing that $\boldsymbol{v} \in X^{\prime} \backslash X$.

First, suppose that $\boldsymbol{w}$ is not matched by $\mathcal{M}_{i}$. Since $\boldsymbol{w} \notin \Gamma_{i}(X)$, this implies that $\boldsymbol{w} \notin \Gamma_{0}(X)$, that is, $\operatorname{dist}(\boldsymbol{w}, X)>M$. On the other hand, every element of $X_{1}$ is at distance at most $M / 2$ from $X$. We conclude that $\boldsymbol{w} \notin X_{1}$. Furthermore, we have $\boldsymbol{w} \in H$ : indeed, $\boldsymbol{w} \in W$ is within distance $M$ from $X^{\prime} \backslash X \subseteq \operatorname{dist}_{\leqslant M}(H)$ so $\operatorname{dist}(\boldsymbol{w}, H) \leqslant 2 M$ and $\boldsymbol{w} \in \mathbb{Z}^{d} \backslash X_{1}$ must belong to $H$. The $\mathcal{M}_{\infty}$-match $\boldsymbol{v}$ of $\boldsymbol{w}$ is at distance at most $M$ from $\boldsymbol{w} \in H$. Thus $\operatorname{dist}(\boldsymbol{v}, H) \leqslant M$ and, by the definition of $X^{\prime}$, we have $\boldsymbol{v} \in X^{\prime}$. Also, $\boldsymbol{v} \notin X$ since $\operatorname{dist}(\boldsymbol{w}, X)>M$. Thus $\boldsymbol{v} \in X^{\prime} \backslash X$, as desired.

So suppose that $\boldsymbol{w}$ is matched by $\mathcal{M}_{i}$. Let $\boldsymbol{u}:=\mathcal{M}_{i}^{-1}(\boldsymbol{w})$. Since $\boldsymbol{w} \in$ $\Gamma_{i}^{\prime}\left(X^{\prime}\right) \subseteq \mathbb{Z}^{d} \backslash \mathcal{M}_{i}^{\prime}(\mathrm{A})$, the edge $(\boldsymbol{u}, \boldsymbol{w}) \in \mathcal{M}_{i}$ was not included into $\mathcal{M}_{i}^{\prime}$. This means by the definition of $\mathcal{M}_{i}^{\prime}$ that $\operatorname{dist}(\boldsymbol{u}, H) \leqslant M$ and thus $\boldsymbol{u} \in X^{\prime}$. Let us derive a contradiction by assuming that $\boldsymbol{v}=\mathcal{M}_{\infty}^{-1}(\boldsymbol{w})$ is different from $\boldsymbol{u}$. So suppose that $\boldsymbol{u} \neq \boldsymbol{v}$. Since $\mathcal{M}_{\infty} \supseteq \mathcal{M}_{i-1}$, we have that $(\boldsymbol{u}, \boldsymbol{w}) \in \mathcal{N}_{i}$. Since $\operatorname{dist}(\boldsymbol{u}, H) \leqslant M$, we have that $(\boldsymbol{u}, \boldsymbol{w})$ is the unique special edge $(\boldsymbol{a}, \boldsymbol{b})$ of $\mathcal{N}_{i}$. However, then $\mathcal{M}_{\infty} \ni(\boldsymbol{a}, \boldsymbol{b})$ maps both $\boldsymbol{u} \neq \boldsymbol{v}$ to the same vertex $\boldsymbol{w}$, a contradiction. Thus $\boldsymbol{v}=\boldsymbol{u}$ is in $X^{\prime}$; also $\boldsymbol{v}=\mathcal{M}_{i}^{-1}(\boldsymbol{w})$ cannot belong to $X \subseteq \mathrm{A}_{i} \subseteq \mathbb{Z}^{d} \backslash \mathcal{M}_{i}^{-1}(\mathrm{~B})$. Thus $\boldsymbol{v} \in X^{\prime} \backslash X$, as desired.

We conclude that $\mathcal{M}_{\infty}^{-1}$ gives an injection from $W$ to $X^{\prime} \backslash X$. This proves Part 5 of the claim.

By Part 5 of Claim 5.2.2, it is enough to prove (33) for $X^{\prime}$ with respect to the smaller matchings $\mathcal{M}_{1}^{\prime} \subseteq \ldots \subseteq \mathcal{M}_{i}^{\prime}$. Note that the assumptions of the lemma cannot be violated by shrinking the matchings, except if $\mathcal{M}_{i}^{\prime}=\emptyset$ 
in which case $\mathcal{M}_{i}^{\prime} \subseteq \mathcal{M}_{i-1}$ is trivially extendable. Also, Parts 3 and 4 of Claim 5.2.2 show that, when we pass from $X$ to $X^{\prime}$, we preserve the $2 M$ connectivity and the set of holes does not change except the non-rich hole $H$ disappears. Thus if we iterate the above operation (that is, keep "filling up" finite non-rich holes one by one until none remains), then we stop in finitely many steps and the final set will satisfy all assumptions of either Case 1 or Case 2.1. Since we gave a direct proof for these cases, this finishes the proof of Lemma 5.2 (and thus of Part 2 of Theorem 2.2).

\section{Concluding remarks}

Laczkovich [21, Page 114] states that "a rough estimate" of the number of pieces for squaring circle given by the proof in $[16,17]$ is $10^{40}$. Since our proof of Theorem 1.2 requires stronger analogues of some inequalities from $[16,17]$, such as the extra term $\Omega\left(|X|^{(d-1) / d}\right)$ in Part 1 of Lemma 4.5 under the further restriction of the neighbourhood to $R$ (whereas the unrestricted bound $|E(X)| \geqslant|X|$ suffices for Theorem 1), it produces at least as many pieces as the proofs by Laczkovich. As mentioned in [21, Page 114], one needs at least 3 pieces for circle squaring with arbitrary isometries and at least 4 pieces if one has to use translations only. This seems still to be the current state of knowledge, so the gap here is really huge.

It is interesting to compare the proofs and results in the current paper and [10] as both give, for example, a Lebesgue measurable version of Hilbert's third problem. In terms of methods, both papers share the same general approach of reducing the problem to finding a measurable matching in a certain infinite bipartite graph $\mathcal{G}=(A, B, E)$, once we have agreed on the exact set of isometries to be used. Like here, the paper [10] constructs a sequence of measurable matchings $\left(\mathcal{M}_{i}\right)_{i \in \mathbb{N}}$ satisfying (13) and (14), and then defines $\mathcal{M}$ by (12). However, the matching $\mathcal{M}_{i}$ in [10] is obtained from $\mathcal{M}_{i-1}$ by augmenting paths of length at most $2 i+1$ in an arbitrary measurable way until none remains. This works by the observation of Lyons and Nazarov [25, Remark 2.6] that (13) and (14) are satisfied automatically provided $\mathcal{G}$ has the expansion property (i.e. there is $\varepsilon>0$ such that the measure of the neighbourhood of $X$ is at least $(1+\varepsilon) \lambda(X)$ for every set $X$ occupying at most half of one part in measure). As shown in [10], the expansion property applies to a wide range of pairs $A, B$. For example, one of the results in [10] is that two bounded Lebesgue measurable sets $A, B \subseteq \mathbb{R}^{k}$ for $k \geqslant 3$ are measurably equidecomposable if $\lambda(A)=\lambda(B)$ and an open ball can be covered by finitely many copies of each set (without any further assumptions on the boundary or interior of these sets). On the other hand, $\mathcal{G}$ cannot have the expansion property if the isometries are taken from an amenable group, for example, such as the group of translations of $\mathbb{R}^{k}$. Thus we could not prove the Lebesgue part of Theorem 1.2 
by doing augmentations as in [10]; instead, we had to carefully guide each $\mathcal{M}_{i}$ to look locally as a binary grid of maximum matchings. Also, various examples by Laczkovich $[16,18,22]$ show that the assumptions of Theorems 1.1-2.2 are rather tight. Hopefully, the ideas that were introduced here will be useful in establishing further results on measurable equidecompositions, in particular under actions of amenable groups.

As far as we see, the only place in this paper where we use any set theoretic assumption stronger than the Axiom of Dependent Choice is the application of Rado's theorem [30] inside Theorem 2.1 to derive the existence of a perfect matching. Thus, if we are allowed to use only the Axiom of Dependent Choice, our proof of Theorem 1.2 should produce a Borel measurable equidecomposition $A \backslash A^{\prime} \stackrel{\operatorname{Tr}}{\sim} B \backslash B^{\prime}$ for some Borel meagre nullsets $A^{\prime} \subseteq A$ and $B^{\prime} \subseteq B$.

Probably, the most interesting problem which remains open is the question of Wagon [40, Page 229] whether circle squaring is possible with Borel pieces. Unfortunately, we do not see a way how to completely eliminate the error set $A^{\prime} \cup B^{\prime}$ (which arises in our arguments after (13)-(14) in Section 4 and after (30) in Section 5), apart from applying Theorem 2.1 and thus using the Axiom of Choice.

\section{Acknowledgements}

The authors are grateful for the helpful comments from Spencer Unger that simplified the proof of Lemma 5.2 and from the anonymous referees that, in particular, improved the bound of Lemma 4.4 .

\section{References}

1. R. Ahlswede and S. L. Bezrukov, Edge isoperimetric theorems for integer point arrays, Appl. Math. Lett. 8 (1995), 75-80.

2. S. Banach, Sur le probléme de la mesure, Fund. Math. 4 (1923), 7-33.

3. S. Banach and A. Tarski, Sur la décomposition des ensembles de points en parties respectivement congruentes., Fund. Math. 6 (1924), 244-277.

4. R. Dougherty and M. Foreman, Banach-Tarski paradox using pieces with the property of Baire, Proc. Nat. Acad. Sci. U.S.A. 89 (1992), 10726-10728.

5. _ Banach-Tarski decompositions using sets with the property of Baire, J. Amer. Math. Soc. 7 (1994), 75-124.

6. L. Dubins, M. W. Hirsch, and J. Karush, Scissor congruence, Israel J. Math. 1 (1963), 239-247.

7. R. J. Gardner, Convex bodies equidecomposable by locally discrete groups of isometries, Mathematika 32 (1985), 1-9.

8. __ Measure theory and some problems in geometry, Atti Sem. Mat. Fis. Univ. Modena 39 (1991), 51-72. 
9. R. J. Gardner and S. Wagon, At long last, the circle has been squared, Notices Amer. Math. Soc. 36 (1989), 1338-1343.

10. Ł. Grabowski, A. Máthé, and O. Pikhurko, Measurable equidecompositions for group actions with an expansion property, E-print arXiv:1601.02958, 2016.

11. E. Hertel and C. Richter, Squaring the circle by dissection, Beiträge Algebra Geom. 44 (2003), 47-55.

12. A. S. Kechris and A. Marks, Descriptive graph combinatorics, Manuscript, 2015.

13. A. S. Kechris and B. D. Miller, Topics in orbit equivalence, Lecture Notes in Mathematics, vol. 1852, Springer, Berlin, 2004.

14. A. S. Kechris, S. Solecki, and S. Todorcevic, Borel chromatic numbers, Adv. Math. 141 (1999), 1-44.

15. M. Laczkovich, Equidecomposability and discrepancy; a solution of Tarski's circlesquaring problem, J. Reine Angew. Math. 404 (1990), 77-117.

16. __ Decomposition of sets with small boundary, J. Lond. Math. Soc. 46 (1992), $58-64$.

17. _ Uniformly spread discrete sets in $\mathbf{R}^{d}$, J. Lond. Math. Soc. 46 (1992), $39-57$.

18. __ Decomposition of sets of small or large boundary, Mathematika 40 (1993), 290-304.

19. __ Equidecomposability of sets, invariant measures, and paradoxes, Rend. Istit. Mat. Univ. Trieste 23 (1993), 145-176, School on Measure Theory and Real Analysis (Grado, 1991).

20. _ Paradoxical decompositions: a survey of recent results, First European Congress of Mathematics, Paris, July 6-10, 1992 (A. Joseph, F. Mignot, F. Murat, B. Prum, and R. Rentschler, eds.), Springer, 1994, pp. 159-184.

21. _ Paradoxes in measure theory, Handbook of measure theory, Vol. I, II (E. Pap, ed.), North-Holland, Amsterdam, 2002, pp. 83-123.

22. __ Equidecomposability of Jordan domains under groups of isometries, Fund. Math. 177 (2003), 151-173.

23. L. H. Loomis and H. Whitney, An inequality related to the isoperimetric inequality, Bull. Amer. Math. Soc. 55 (1949), 961-962.

24. L. Lovász, Large networks and graph limits, Colloquium Publications, Amer. Math. Soc., 2012.

25. R. Lyons and F. Nazarov, Perfect matchings as IID factors on non-amenable groups, European J. Combin. 32 (2011), 1115-1125.

26. A. Marks and S. Unger, Baire measurable paradoxical decompositions via matchings, Adv. Math. 289 (2016), 397-410.

27. J. von Neumann, Zur allgemeinen Theorie des Masses, Fund. Math. 13 (1929), $73-116$. 
28. H. Niederreiter and J. M. Wills, Diskrepanz und Distanz von Maßen bezüglich konvexer und Jordanscher Mengen, Math. Z. 144 (1975), 125-134.

29. J. C. Oxtoby, Measure and category, second ed., Graduate Texts in Mathematics, vol. 2, Springer-Verlag, New York, 1980.

30. R. Rado, Factorization of even graphs, Quart. J. Math., Oxford Ser. 20 (1949), 95-104.

31. C. Richter, Congruence by dissection of topological discs - an elementary approach to Tarski's circle squaring problem, Discrete Comput. Geom. 28 (2002), 427-442.

32. __ Affine congruence by dissection of discs - appropriate groups and optimal dissections, J. Geom. 84 (2005), 117-132.

33. __ Squaring the circle via affine congruence by dissection with smooth pieces, Beiträge Algebra Geom. 48 (2007), 423-434.

34. A. Tarski, O równowazńosći wielokatów (in Polish, with French summary), Przeglad Matematyczno-Fizyczny 1-2 (1924), 54.

35. _ Problème 38, Fund. Math. 7 (1925), 381.

36. Á. Timár, Tree and grid factors for general point processes, Electron. Comm. Probab. 9 (2004), 53-59 (electronic).

37. _ Invariant matchings of exponential tail on coin flips in $Z^{d}$, E-print arxiv:0909.1090, 2009.

38. G. Tomkowicz and S. Wagon, The Banach-Tarski paradox, 2d ed., Cambridge University Press, Cambridge, 2016, With a foreword by Jan Mycielski.

39. S. Wagon, Circle-squaring in the twentieth century, Math. Intelligencer 3 (1980/81), 176-181.

40. _ The Banach-Tarski paradox, Cambridge University Press, Cambridge, 1993, With a foreword by Jan Mycielski, corrected reprint of the 1985 original.

(Received: December 3, 2015)

(Revised: August 2, 2016)

Department of Mathematics and Statistics, Lancaster University, Lancaster, UNITED KInGDOM

E-mail: graboluk@gmail.com

Mathematics Institute, University of Warwick, Coventry, United Kingdom

E-mail: A.Mathe@warwick.ac.uk

Mathematics Institute and DimAP, University of Warwick, Coventry, United KINGDOM

E-mail: O.Pikhurko@warwick.ac.uk 